\title{
X-ray emission from the young brown dwarfs of the Taurus molecular cloud ${ }^{\star}$
}

\author{
N. Grosso ${ }^{1}$, K. R. Briggs ${ }^{2}$, M. Güdel ${ }^{2}$, S. Guieu ${ }^{1}$, E. Franciosini ${ }^{3}$, F. Palla ${ }^{4}$, \\ C. Dougados ${ }^{1}$, J.-L. Monin ${ }^{1,5}$, F. Ménard ${ }^{1}$, J. Bouvier ${ }^{1}$, M. Audard ${ }^{6}$, and A. Telleschi ${ }^{2}$ \\ ${ }^{1}$ Laboratoire d'Astrophysique de Grenoble, Université Joseph-Fourier, 38041 Grenoble Cedex 9, France \\ e-mail: Nicolas.Grosso@obs.ujf-grenoble.fr \\ 2 Paul Scherrer Institut, 5232 Villigen und Würenlingen, Switzerland \\ INAF - Osservatorio Astronomico di Palermo, Piazza del Parlamento 1, 90134 Palermo, Italy \\ INAF - Osservatorio Astrofisico di Arcetri, Largo Enrico Fermi 5, 50125 Firenze, Italy \\ Institut Universitaire de France \\ ${ }^{6}$ Columbia Astrophysics Laboratory, Columbia University, 550 West 120th Street, New York, NY 10027, USA \\ Received 5 May 2006 / Accepted 5 August 2006
}

\section{ABSTRACT}

\begin{abstract}
Aims. We report the X-ray properties of young ( 3 Myr) bona fide brown dwarfs of the Taurus Molecular Cloud (TMC).
Methods. The XMM-Newton Extended Survey of the TMC (XEST) is a large program designed to systematically investigate the X-ray properties of young stellar/substellar objects in the TMC. In particular, the area surveyed by 15 XMM-Newton pointings (of which three are archival observations), supplemented with one archival Chandra observation, allows us to study 17 brown dwarfs with $\mathrm{M}$ spectral types.

Results. Half of this sample ( 9 out of 17 brown dwarfs) is detected; 7 brown dwarfs are detected here for the first time in X-rays. We observed a flare from one brown dwarf. We confirm several previous findings on brown dwarf X-ray activity: a log-log relation between X-ray and bolometric luminosity for stars (with $L_{*} \leq 10 L_{\odot}$ ) and brown dwarfs detected in X-rays, which is consistent with a mean X-ray fractional luminosity $\left\langle\log \left(L_{\mathrm{X}} / L_{*}\right)\right\rangle=-3.5 \pm 0.4$; for the XEST brown dwarfs, the median of $\log \left(L_{\mathrm{X}} / L_{*}\right)$ (including upper limits) is -4.0 ; a shallow log-log relation between X-ray fractional luminosity and mass; a log-log relation between X-ray fractional luminosity and effective temperature; a log-log relation between X-ray surface flux and effective temperature. We find no significant $\log -\log$ correlation between the X-ray fractional luminosity and $E W(\mathrm{H} \alpha)$. Accreting and nonaccreting brown dwarfs have a similar $\mathrm{X}$-ray fractional luminosity. The median X-ray fractional luminosity of nonaccreting brown dwarfs is about 4 times lower than the mean saturation value for rapidly rotating low-mass field stars. Our TMC brown dwarfs have higher X-ray fractional luminosity than brown dwarfs in the Chandra Orion Ultradeep Project.

Conclusions. The X-ray fractional luminosity declines from low-mass stars to M-type brown dwarfs, and as a sample, the brown dwarfs are less efficient X-ray emitters than low-mass stars. We thus conclude that while the brown dwarf atmospheres observed here are mostly warm enough to sustain coronal activity, a trend is seen that may indicate its gradual decline due to the drop in photospheric ionization degree.
\end{abstract}

Key words. stars: low-mass, brown dwarfs - X-rays: stars - ISM: individual objects: Taurus molecular cloud

\section{Introduction}

Pre-main-sequence low-mass stars, i.e. T Tauri stars, show a high level of X-ray emission, which is generally attributed to an active corona, an enhanced version of the magnetic corona on the contemporary Sun (see for a review Feigelson \& Montmerle 1999). The dynamo mechanism producing the magnetic field in these fully convective stars is still discussed (Preibisch et al. 2005a). $\mathrm{X}$-ray observations of brown dwarfs (BDs) allow investigation of the magnetic activity in the substellar regime, where masses are lower than about $0.075 M_{\odot}$.

The first X-ray detection of a BD was reported by Neuhäuser \& Comerón (1998), who identified in the Cha I dark cloud an X-ray source detected by ROSAT - in early 1991, before the near-infrared observations of the first BDs were reported (Nakajima et al. 1995; Rebolo et al. 1995) - as the counterpart

^ Appendices A, B, C, are only available in electronic form at http://www . aanda. org of a young BD. ROSAT detected only a few BDs or very lowmass stars, all of them young and located in star-forming regions (Neuhäuser et al. 1999; Comerón et al. 2000; Mokler \& Stelzer 2002).

The new generation of X-ray satellites, XMM-Newton and Chandra, allows now detection of more BDs thanks to their increase of sensitivity. The number of young or intermediate-age BDs detected in X-rays (e.g., Imanishi et al. 2001; Preibisch \& Zinnecker 2001, 2002; Tsuboi et al. 2003; Briggs \& Pye 2004; Stelzer et al. 2004; Ozawa et al. 2005; Stelzer et al. 2006) exceeds largely the one of (older) field BDs, which have been detected so far mainly during X-ray flares (Rutledge et al. 2000; Stelzer 2004). Recently, the X-ray properties of 33 young BDs (Slesnick et al. 2004) in the Orion Nebula Cluster (ONC) located at $\sim 450 \mathrm{pc}$ were studied by Preibisch et al. (2005b) in the Chandra Orion Ultradeep Project (COUP).

We investigate in this paper the X-ray properties of a sample of 17 BDs in the nearby $(140 \mathrm{pc})$ Taurus Molecular Cloud 
(TMC), surveyed in the XMM-Newton Extended Survey of the TMC (XEST; Güdel et al. 2007). First, we define in Sect. 2 the TMC BD sample, review previous X-ray results on it, and report the BDs detected in the XEST. Analysis of the X-ray variability of the TMC BDs is presented in Sect. 3, and X-ray spectral properties are determined in Sect. 4. In Sect. 6, we compare the $\mathrm{X}$-ray luminosities of the BDs and low-mass stars observed in the XEST. Comparison of the X-ray fractional luminosities of BDs in the XEST and COUP is made in Sect. 7. We discuss the origin of the BD X-ray emission in the broader context of the $\mathrm{X}$-ray emission of cool stars in Sect. 8. Finally, we summarize our results in Sect. 9.

\section{The TMC brown dwarf sample}

For a typical TMC member, with an age of $3 \mathrm{Myr}$, the stellar/substellar boundary at $0.075 M_{\odot}$ is between M6V and M6.5V according to pre-main-sequence tracks from Baraffe et al. (1998). Therefore, we compiled from the literature objects with spectral type later than M6V to build our bona fide BD sample ${ }^{1}$.

The first optical imaging and spectroscopic surveys of BDs in the TMC concentrated on high stellar density regions, while the majority of the volume occupied by the molecular clouds was left unexplored (Briceño et al. 1998; Luhman 2000; Briceño et al. 2002; Luhman 2004). Recently, Guieu et al. (2006) performed a large scale optical imaging survey of TMC with the Canada-France-Hawaii telescope with the CFH12k and MEGACAM large-scale optical cameras, covering a total area of $\approx 28 \mathrm{deg}^{2}$, and encompassing the densest parts of the cloud as well as their surroundings. By employing all-sky catalogs, Luhman (2006) considered a $15^{\circ} \times 15^{\circ}$ area, large enough to encompass all of the TMC. These optical surveys of the TMC, conducted since nearly 10 years, yielded the identification of 42 BDs.

\subsection{Previous $X$-ray results on the TMC brown dwarfs}

Two BDs of this sample were previously detected in X-rays by the ROSAT/Position Sensitive Proportional Counter (PSPC) which was sensitive only to soft X-rays from 0.1 to $2.4 \mathrm{keV}-$ but without being identified as substellar objects.

The BD MHO 4 (Briceño et al. 1998; Luhman 2000) was first detected during a $4.0 \mathrm{ks}$ ROSAT/PSPC pointed observation of V826 Tau in August 1992 with an X-ray count rate of 4 counts $\mathrm{ks}^{-1}$ and proposed as a new weak-line T Tauri star of Taurus-Auriga (source RXJ0431.3+1800 of Wichmann et al. 1996). MHO 4 was also detected during a $7.7 \mathrm{ks}$ ROSAT/PSPC pointed observation of the dark cloud Lynds 1551 in February 1993 with an X-ray count rate of 3.1 counts ks $^{-1}$ and proposed as a new member of the TMC (source L1551X 15 of Carkner et al. 1996, associated with a "faint, very red star"). Neuhäuser et al. (1999) reported these two X-ray detections of MHO 4, considered at that time as a BD candidate ${ }^{2}$.

${ }^{1}$ In XEST, there are only five objects with a M6V spectral type (namely, V410 X-ray 3, MHO 5, MHO 8, KPNO-Tau 14, and CFHT-Tau 12), which have luminosities higher than $0.03 L_{\odot}$, placing them above the stellar/substellar boundary (Baraffe et al. 1998).

2 V410 Anon 13, V410 X-ray 3, and MHO 5, which were considered as BD candidates detected in X-rays by ROSAT (Neuhäuser et al. 1999), have now more reliable spectral types (M5.75V, M6V, and M6V, respectively; Briceño et al. 2002), which combined with their luminosities place them above the stellar/substellar boundary. Therefore these very low-mass stars were not included in our sample of BDs. However, they were detected in the XEST and will hence be used in Sect. 5 as TMC low-mass stars detected in X-rays.
CFHT-BD-Tau 4, one of the BDs of Martín et al. (2001), was detected during a $10.6 \mathrm{ks}$ ROSAT/PSPC pointed observation of the Heiles dark cloud 2 NW in March 1993 with an X-ray count rate of 4.5 counts ks ${ }^{-1}$ corresponding to a luminosity of $\sim 10^{29} \mathrm{erg} \mathrm{s}^{-1}$ (source HCL $2 \mathrm{NW}-7$ a of Briceño et al. 1999, with no optical counterpart but a NIR counterpart). Mokler \& Stelzer (2002) reported this X-ray source as the counterpart of this BD.

The higher sensitivity of the new generation of X-ray satellites, XMM-Newton (Jansen et al. 2001) and Chandra (Weisskopf et al. 2002), allows detection of BDs which are from $\sim 10$ to $\sim 100$ times fainter in X-rays.

\subsection{X-ray detected brown dwarfs in the XEST}

The XMM-Newton Extended Survey of the TMC (XEST) is a large program designed to systematically investigate the highenergy properties of young stellar and substellar objects in this nearest star-forming region. It is composed of 19 dedicated $X M M-N e w t o n$ pointings plus 8 archival XMM-Newton observations. The total $X M M-N e w t o n$ exposure time is about $300 \mathrm{~h}$ shared among these 27 pointings (Güdel et al. 2007). We will focus here on the X-ray detection of BDs with the three X-ray telescopes of XMM-Newton - two European Photon Imaging Cameras (EPIC) equipped with MOS CCD arrays (Turner et al. 2001), and the third carrying a pn CCD camera (Strüder et al. 2001) - simultaneous $U$-band observations obtained with the $X M M-N e w t o n$ optical/UV monitor are discussed in Grosso et al. (2007).

Figure 1 shows the XEST area and the locations of the 42 TMC BDs. Fifteen XMM-Newtonpointings (of which three are archival observations) surveyed 16 BDs. We looked also for serendipitous Chandraobservations at the Chandra X-ray Center's archives (see online Appendix A, Table A.1). We supplemented the XEST with the ACIS-I observation of L1527 which allow us to survey and detect one more BD (CFHT-BDTau 4); and with the ACIS-I observation of L1551 which provides a second epoch observation of MHO 4. Therefore, we surveyed in X-rays 17 out of the 42 TMC BDs, which allows us to survey the X-ray emission of $40 \%$ of the known BDs in the TMC.

Table 1 lists the properties of these 17 BDs. Figure 2 gives their location in the HR diagram. The coolest object of the surveyed $\mathrm{BD}$ sample has a spectral type $\mathrm{M} 9.5 \mathrm{~V}$, corresponding to about $0.02 M_{\odot}$ (Baraffe et al. 2002). Their luminosities range from $0.075 L_{\odot}$ to $0.001 L_{\odot}$. The optical extinctions of this sample range from a negligible value to about 9 mag with a mean and a median of 1.5 and $0.5 \mathrm{mag}$, respectively. The average low extinction of this sample is mainly due to the initial selection process for TMC candidate BDs based on optical photometry, and therefore biased towards low extinction BDs.

The list of X-ray sources in the XEST (Güdel et al. 2005) was completed with X-ray sources of the two archival Chandra observations (see online Appendix A for details on the data reduction of Chandra data). We cross-correlated the BD positions with the positions of all X-ray sources. We detected 9 BDs in X-rays (see Table 2). The two BDs previously detected with ROSAT were redetected with XMM-Newton and Chandra. The detection of MHO 4 in L1551 was previously published by Favata et al. (2003) with XMM-Newton, and Bally et al. (2003) with Chandra. Seven BDs are detected here for the first time in $\mathrm{X}$-rays. The detection rate in X-rays of these $17 \mathrm{BDs}$ is therefore $53 \%$.

We detected mainly the BDs with luminosities greater than $\sim 0.01 L_{\odot}$, i.e. the BDs with spectral type earlier or equal than M8V (Fig. 2). 


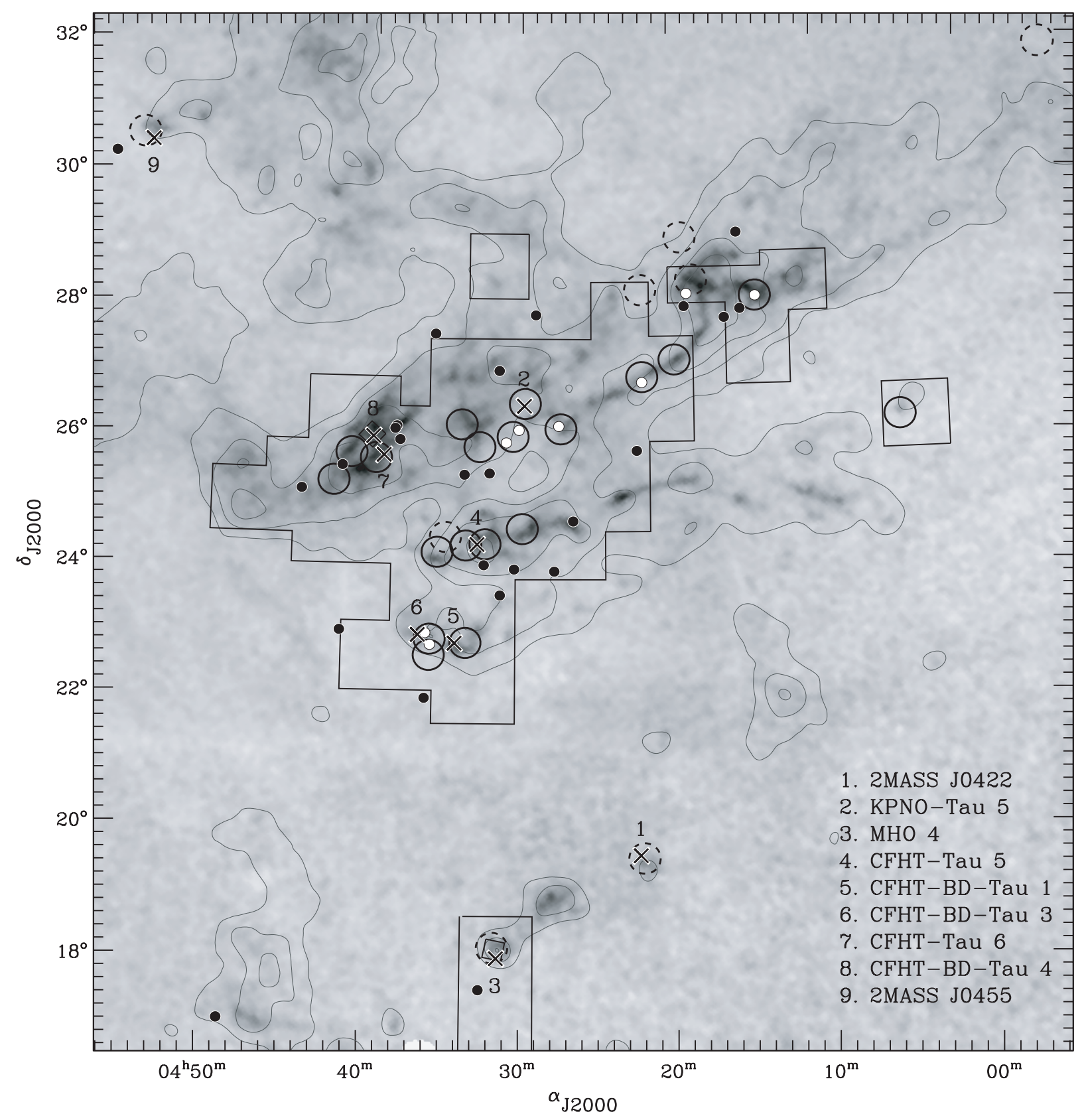

Fig. 1. The XMM-Newton Extended Survey of the Taurus Molecular Cloud (XEST). Contours show the ${ }^{12}$ CO emission (Dame et al. 1987) of the Taurus Molecular Cloud (TMC), overlaid on a visual extinction map (linear colour scale, with black colour corresponding to $A_{\mathrm{V}} \sim 6$ mag; based on DSS I, 6'-resolution map, Dobashi et al. 2005). The 27 XMM-Newton fields of view of the XEST are plotted with continuous/dashed circles (of which seven are archival observations and one is a separate program on T Tau; dashed circles). Note the outlying XMM-Newton fields of view around SU Aur (NE corner) and L1551 (S). The two squares near labels 3 and 8 show the archival Chandra fields of view used in this work (see Table A.1). The continuous line shows the survey of brown dwarfs (BDs) performed with the Canada-France-Hawaii telescope (Guieu et al. 2006). This region hosts 42 young BDs of the TMC (Briceño et al. 1998, 2002; Luhman 2000, 2004, 2006; Martín et al. 2001; Guieu et al. 2006). Black dots indicate the 25 BDs of this region which were not surveyed in X-rays with the XEST. White dots show the 8 BDs not detected in X-rays. Crosses show the 9 BDs detected in X-rays (Table 2) with labeled numbers referring to BD names. Only 2 BDs of TMC were previously detected in X-rays by ROSAT: MHO 4 (Carkner et al. 1996; Neuhäuser et al. 1999) and CFHT-BD-Tau 4 (Briceño et al. 1999; Mokler \& Stelzer 2002).

\section{X-ray variability analysis of the TMC brown dwarfs}

Obvious variability was observed only from CFHT-BD-Tau 1, a nonaccreting M7V BD, which displayed an X-ray (coronal) flare during $\sim 30 \%$ of the observation period (Fig. 3). The shape of this light curve, a fast rise followed by exponential decay, is typical of X-ray flares from young stellar objects (e.g., Imanishi et al. 2003; Favata et al. 2005). We fit the non-zero count rates with a quiescent level plus an exponential rise and decay (Fig. 3). We estimate from this light curve fit that the quiescent level contributes to $54 \%$ of the total counts (quiescent+flare) detected from CFHT-BD-Tau 1. The quiescent emission level is $1.8 \pm 0.5$ counts ks${ }^{-1}$, the flare amplitude is $13.3 \pm 2.9$ counts ks ${ }^{-1}$, i.e. about 8 times the quiescent level. The time scales of the rise and decay phase are $\tau_{\text {rise }}=15 \pm 5 \mathrm{~min}$ and $\tau_{\text {decay }}=47 \pm 11 \mathrm{~min}$, respectively. Both values are in the lower range of time scales observed in the systematic study of X-ray flares from low-mass 
Table 1. The sample of the 17 young BDs surveyed in the XEST. Column (2) gives the reference of the discovery paper. Col. (3) give the 2MASS counterparts. Column (7) gives the references for the spectral type (Col. 4), the optical extinction (when not available derived from $A_{\mathrm{J}}$ using Rieke \& Lebofsky 1985, Col. 5), and the reference of the equivalent width of $\mathrm{H} \alpha(E W(\mathrm{H} \alpha)$, given in Col. 6) if it is different. Negligible optical extinction in Col. (5) are indicated by 0. The effective temperature in Col. (8) has been computed from the spectral type using the temperature scale $T_{\text {eff }}=3841.94-141.17 \times$ SpTyp, which is valid for young stars with M spectral type (Guieu et al. 2006). The visual extinction in Col. (5) is taken when available from the literature; for 2MASS J0421 and 2MASS J0422, we averaged the visual extinction computed from $J-H, H-K_{\mathrm{S}}$, and the spectral type, using a dwarf sequence (compiled from the literature; e.g., Leggett et al. 1998). The bolometric luminosity of the substellar photosphere in Col. (9) has been computed from $I, J$-band magnitudes and $A_{\mathrm{V}}$ (see Guieu et al. 2006); for 2MASS J0414 and MHO 4 (without I-band magnitude available) the reference for the luminosity is Luhman (2004) and Briceño et al. (2002), respectively. Column (10) indicates accreting sources based mainly on $E W(\mathrm{H} \alpha)$ (see Sect. 6.2). The last column indicates detection in X-rays (this work; see Table 2). References: B98 = Briceño et al. (1998); B02 = Briceño et al. (2002); G06 = Guieu et al. (2006); L04 = Luhman (2004); L06 = Luhman (2006); M01 = Martín et al. (2001); M05 = Muzerolle et al. (2005a).

\begin{tabular}{|c|c|c|c|c|c|c|c|c|c|c|}
\hline Name & Ref. & 2MASS & SpTyp & $\begin{array}{c}A_{\mathrm{V}} \\
\mathrm{mag} \\
(5)\end{array}$ & $\begin{array}{c}E W(\mathrm{H} \alpha) \\
\AA \\
(6)\end{array}$ & Ref. & $\begin{array}{c}T_{\text {eff }} \\
\mathrm{K} \\
(8)\end{array}$ & $\begin{array}{l}L_{*} \\
L_{\odot} \\
(9)\end{array}$ & Acc. & (11) \\
\hline 2MASS J0414 & L04 & J04141188+2811535 & M6.25 & 1.1 & 250.0 & M05 & 2960 & 0.015 & $\mathrm{y}$ & $\mathrm{n}$ \\
\hline KPNO-Tau 2 & B02 & J04185115+2814332 & M6.75 & 0.4 & 8.4 & G06 & 2889 & 0.007 & $\mathrm{n}$ & $\mathrm{n}$ \\
\hline 2MASS J0421 & L06 & $\mathrm{J} 04215450+2652315$ & M8.5 & 3.0 & & L06 & 2642 & 0.003 & & $\mathrm{n}$ \\
\hline 2MASS J0422 & L06 & J04221332+1934392 & M8 & 1.0 & & L06 & 2713 & 0.017 & & $\mathrm{y}$ \\
\hline KPNO-Tau 4 & B02 & $\mathrm{J} 04272799+2612052$ & M9.5 & 2.5 & 158.1 & G06 & 2501 & 0.004 & $\mathrm{y}$ & $\mathrm{n}$ \\
\hline KPNO-Tau 5 & B02 & $\mathrm{J} 04294568+2630468$ & M 7.5 & 0 & 30.0 & B02 & 2783 & 0.023 & $\mathrm{n}$ & $\mathrm{y}$ \\
\hline KPNO-Tau 6 & B02 & J04300724+2608207 & M9 & 0.9 & 207.9 & G06 & 2571 & 0.003 & $\mathrm{y}$ & $\mathrm{n}$ \\
\hline KPNO-Tau 7 & B02 & J04305718+2556394 & M 8.25 & 0 & 300.0 & B02 & 2677 & 0.004 & $\mathrm{y}$ & $\mathrm{n}$ \\
\hline MHO 4 & B98 & J04312405+1800215 & M7 & 0.5 & 42.0 & B02 & 2854 & 0.048 & $\mathrm{n}$ & $\mathrm{y}$ \\
\hline CFHT-Tau 5 & G06 & J04325026+2422115 & M 7.5 & 9.2 & 29.8 & G06 & 2783 & 0.075 & $\mathrm{n}$ & $\mathrm{y}$ \\
\hline CFHT-BD-Tau 1 & M01 & J04341527+2250309 & M7 & 3.1 & 19.0 & M01 & 2854 & 0.017 & $\mathrm{n}$ & $\mathrm{y}$ \\
\hline KPNO-Tau 9 & B02 & J04355143+2249119 & M 8.5 & 0 & 20.0 & B02 & 2642 & 0.001 & $\mathrm{n}$ & $\mathrm{n}$ \\
\hline CFHT-BD-Tau 2 & M01 & J04361038+2259560 & M 7.5 & 0 & 13.0 & B02 & 2783 & 0.007 & $\mathrm{n}$ & $\mathrm{n}$ \\
\hline CFHT-BD-Tau 3 & M01 & J04363893+2258119 & M 7.75 & 0 & 55.0 & B02 & 2747 & 0.007 & $\mathrm{n}$ & $\mathrm{y}$ \\
\hline CFHT-Tau 6 & G06 & J04390396+2544264 & M 7.25 & 0.4 & 63.7 & G06 & 2818 & 0.024 & $\mathrm{y}$ & $\mathrm{y}$ \\
\hline CFHT-BD-Tau 4 & M01 & J04394748+2601407 & M7 & 2.6 & 340.0 & L04, M01 & 2854 & 0.062 & $\mathrm{y}$ & $\mathrm{y}$ \\
\hline 2MASS J0455 & L04 & $\mathrm{J} 04552333+3027366$ & M 6.25 & 0 & $\ldots \ldots \ldots$ & L04 & 2960 & 0.015 & $\ldots$ & $\mathrm{y}$ \\
\hline
\end{tabular}

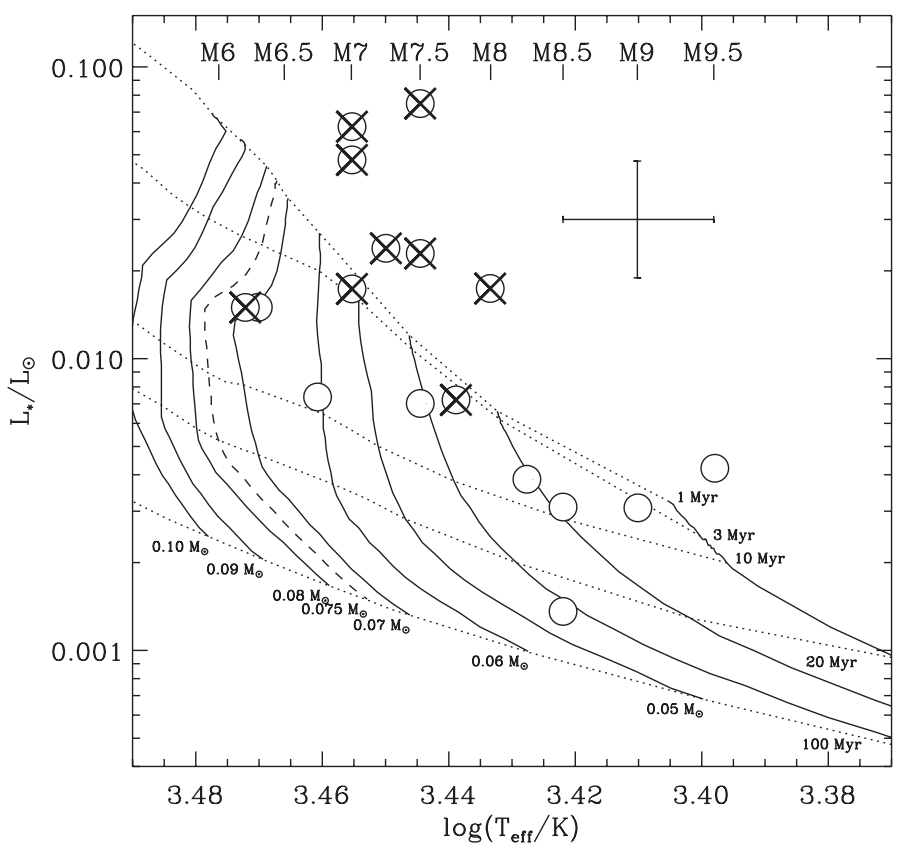

Fig. 2. HR diagram of the 17 BDs of the TMC surveyed in the XEST. The references for the computation of the effective temperatures and the luminosities are given in Table 1 . The pre-main-sequence tracks from Baraffe et al. (1998) are shown for comparison. Continuous lines show mass tracks from 0.1 down to $0.02 M_{\odot}$ in steps of $0.01 M_{\odot}$; the dashed line indicates the stellar/substellar boundary at $0.075 M_{\odot}$, which is equivalent to spectral type later than M6V for a typical TMC member age of 3 Myr. A cross indicates typical uncertainties. Two circles have been slightly moved in spectral type to avoid overlaps. The 9 BDs detected in X-rays are marked with "X".

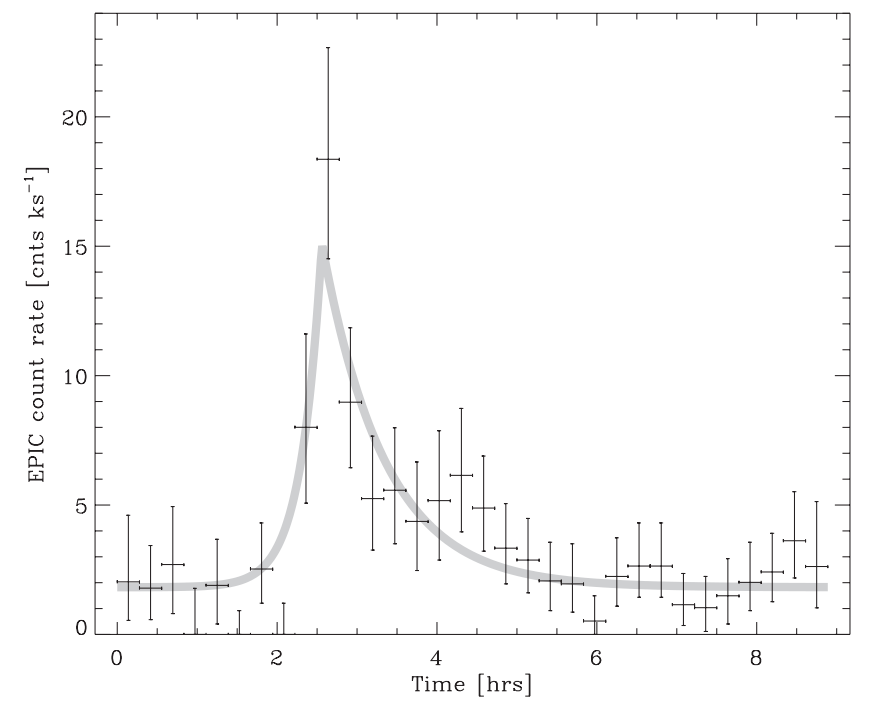

Fig. 3. Light curve of the X-ray flare from CFHT-BD-Tau 1. The bin size is $1000 \mathrm{~s}$. The grey thick line shows the fit of the non-zero count rates using a quiescent level plus an exponential rise and decay.

young stellar objects in the $\rho$ Ophiuchi star-forming region with Chandra (Imanishi et al. 2003). In this latter star-forming region, Ozawa et al. (2005) observed with XMM-Newton from GY310, a young M8.5V BD (Wilking et al. 1999), an X-ray flare with a similar amplitude (peaking 6 times above the quiescent level) and decay time scale, but with a rise phase about 5 times longer. Assuming that the count rate scales linearly with the X-ray luminosity (e.g., Mitra-Kraev et al. 2005), we compute, from the 

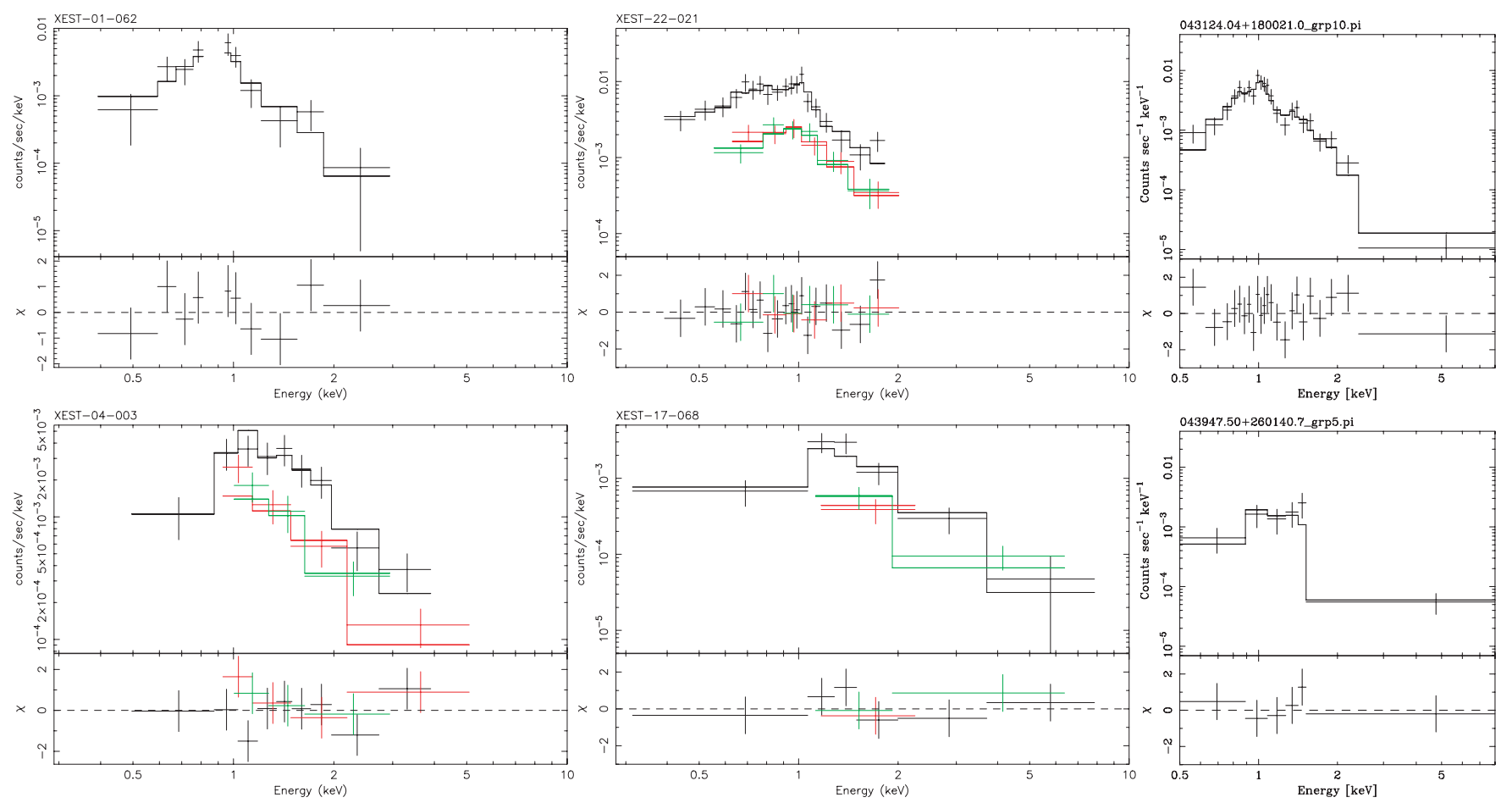

Fig. 4. X-ray spectra of TMC BDs. From top to bottom and left to right: 2MASS J0422 (pn spectrum), MHO 4 (XMM-Newton and Chandra spectra), CFHT-Tau 5 (XMM-Newton spectra), CFHT-BD-Tau 1 (XMM-Newton spectra), CFHT-BD-Tau 4 (Chandra spectra). Black, red, and green code for XMM-Newton/EPIC pn, MOS1, and MOS2 spectra, respectively. The continuous lines show our best fits obtained with an absorbed single-temperature or two-temperature (MHO 4 spectra) plasma model (see Table 3).

average X-ray luminosity of CFHT-BD-Tau 1 (see Table 3), that the total energy released by the CFHT-BD-Tau 1 flare is about $2 \times 10^{33} \mathrm{erg}$.

The X-ray light curves of the other BDs show no large variability (Fig. B.1). Two BDs, observed at two differents epochs, show variations in X-ray luminosity lower than a factor of 2 (Fig. B.2; see below Tables 3 and 4). We looked for a possible modulation of the EPIC light curve of MHO 4 (see Fig. B.2) using a Lomb-Scargle periodogram (e.g., Flaccomio et al. 2005), but we found no statistically significant periodic signal. The BD count rates observed in the XEST are mainly representative of a "quiescent" emission, however (micro)flaring activity could be present even though unresolved in the light curves as observed in active stars (Güdel et al. 2003).

\section{X-ray spectral properties of the TMC brown dwarfs}

\section{1. $X$-ray spectra of the brightest $X$-ray sources}

In total, we have 11 X-ray observations of the 9 BDs detected in the XEST (Table 2). We performed spectral fits for the two Chandra sources, and for these XMM-Newton sources with more than $\sim 100$ counts (Fig. 4). The computation of $X M M$-Newton source spectra with associated redistribution matrix files (RMFs) and auxiliary response files (ARFs) is detailed in Güdel et al. (2007), for Chandra observations see online Appendix A. Spectral fitting was performed with XSPEC (version 11.3; Arnaud 1996) using one or two optically thin thermal plasma models (MEKAL; Mewe et al. 1995) with 0.3 times the solar elemental abundances, combined with an X-ray absorption model (WABS; Morrison \& McCammon 1983), i.e. the same model as used in COUP to allow us to directly compare with the result from Preibisch et al. (2005b). For XMM-Newton spectra, we fitted simultaneously the EPIC pn, MOS1, and MOS2 spectra.

Table 3 gives our best fit parameters obtained for 4 BDs in $3 X M M$-Newton and 2 Chandra observations. The XMMNewton and Chandra spectra of the brightest source, MHO 4, are better described with a two-temperature plasma. The two plasma temperatures found separately from XMM-Newton and Chandra spectra are consistent with each other. For CFHT-Tau 5, the hydrogen column density, $N_{\mathrm{H}}$, determined by one temperature spectral fitting is 3 (with an acceptable range of 2-4) times lower than the value predicted from $A_{\mathrm{V}}=9.2 \pm 0.8 \mathrm{mag}$ (Guieu et al. 2006) using the $N_{\mathrm{H}} / A_{\mathrm{J}}$ ratio of Vuong et al. (2003) combined with the $A_{\mathrm{V}} / A_{\mathrm{J}}$ ratio of Cardelli et al. (1989) for $R_{\mathrm{V}}=3.1$, which leads to $N_{\mathrm{H}}=1.6 \times 10^{21} A_{\mathrm{V}} \mathrm{cm}^{-2} \mathrm{mag}^{-1}$. This may point out a non-canonical dust-to-gas ratio on the line of sight towards this object.

\subsection{Quantile analysis of the faintest $X$-ray sources}

For the faintest X-ray sources, we used a quantile analysis, the new spectral classification technique for X-ray sources proposed by Hong et al. (2004). This technique uses a quantile diagram based on the X-ray colours: $x \equiv \log \left(Q_{50} /\left(1-Q_{50}\right)\right)$, and $y \equiv 3 \times$ $Q_{75} / Q_{25}$, where $Q_{\mathrm{x}} \equiv\left(E_{\mathrm{x} \%}-E_{\mathrm{i}}\right) /\left(E_{\mathrm{f}}-E_{\mathrm{i}}\right)$, with $E_{\mathrm{x}} \%$ the energy where the net counts are $\mathrm{x} \%$ of the source net counts in the $E_{\mathrm{i}}-E_{\mathrm{f}}$ energy range. We take here $E_{\mathrm{i}}=0.5 \mathrm{keV}$ and $E_{\mathrm{f}}=7.3 \mathrm{keV}$.

With the spectral model of Sect. 4.1, we calculated for pn and MOS1+MOS2, from the Perl script provided by Hong et al. $(2004)^{3}$, a grid of hydrogen column densities and plasma

\footnotetext{
${ }^{3}$ The version 1.7 of the IDL and Perl quantile analysis softwares of Hong et al. (2004) is available at
} http://hea-www. harvard. edu/ChaMPlane/quantile 
Table 2. Detection list of young BDs in the XEST. Column (1) numbers correspond to labels in Fig. 1. Columns (2)-(4) give the BD, satellite and target names, respectively. The naming of XMM-Newton sources in Col. (5) follows the convention of Güdel et al. (2007), where the two and three digits code for the field and the source number in this field, respectively. Columns (6)-(9) give X-ray source positions, total positional uncertainties, and distance to the 2MASS position, respectively. References: [FGMSD03] = Favata et al. $(2003)$, [BFR03] = Bally et al. $(2003)$. There is only pn data for X-ray source \#1 as it falls in the gap of the MOS CCDs in window mode in this observation. X-ray source \#4b is affected by pn gap. For X-ray source \#9 only MOS data are available.

\begin{tabular}{|c|c|c|c|c|c|c|c|c|}
\hline $\begin{array}{c}\# \\
(1)\end{array}$ & $\begin{array}{c}\text { BD name } \\
\text { (2) }\end{array}$ & $\begin{array}{c}\text { Satellite } \\
\text { (3) }\end{array}$ & $\begin{array}{c}\text { Target } \\
\text { (4) }\end{array}$ & X-ray source name & $\begin{array}{c}\alpha_{\mathrm{J} 2000} \\
(6)\end{array}$ & $\begin{array}{c}\delta_{\mathrm{J} 2000} \\
(7)\end{array}$ & $\begin{array}{c}\text { err. } \\
\prime \prime \\
(8)\end{array}$ & $\begin{array}{c}\text { dist. } \\
\text { " } \\
\text { (9) }\end{array}$ \\
\hline 1 & 2MASS J0422 & XMM & T Tau & XEST-01-062 & $04^{\mathrm{h}} 22^{\mathrm{m}} 13^{\mathrm{s}} .2$ & $19^{\circ} 34^{\prime} 40^{\prime} 2$ & 1.7 & 1.5 \\
\hline 2 & KPNO-Tau 5 & $\mathrm{XMM}$ & DI Tau & XEST-15-044 & $04^{\mathrm{h}} 29^{\mathrm{m}} 45^{\mathrm{s}} .8$ & $26^{\circ} 30^{\prime} 47^{\prime \prime} 9$ & 1.8 & 1.9 \\
\hline $3 \mathrm{a}$ & $\mathrm{MHO} 4$ & $\mathrm{XMM}$ & V955 Tau & XEST-22-021 = [FGMSD03] 17 & $04^{\mathrm{h}} 31^{\mathrm{m}} 24^{\mathrm{s}} .2$ & $18^{\circ} 00^{\prime} 21^{\prime \prime} 6$ & 1.6 & 2.1 \\
\hline $3 b$ & MHO 4 & $\mathrm{CXO}$ & L1551 & [BFR03] 18 & $04^{\mathrm{h}} 31^{\mathrm{m}} 24^{\mathrm{s}} .1$ & $18^{\circ} 00^{\prime} 21^{\prime \prime} 4$ & 0.7 & 0.7 \\
\hline $4 \mathrm{a}$ & CFHT-Tau 5 & XMM & GK Tau & XEST-04-003 & $04^{\mathrm{h}} 32^{\mathrm{m}} 50^{\mathrm{s}} \cdot 3$ & $24^{\circ} 22^{\prime} 11^{\prime \prime} .1$ & 1.7 & 0.7 \\
\hline $4 \mathrm{~b}$ & CFHT-Tau 5 & XMM & V928 Tau & XEST-03-031 & $04^{\mathrm{h}} 32^{\mathrm{m}} 50^{\mathrm{s}} \cdot 3$ & $24^{\circ} 22^{\prime} 11^{\prime \prime} .4$ & 1.7 & 0.6 \\
\hline 5 & CFHT-BD-Tau 1 & XMM & CI Tau & XEST-17-068 & $04^{\mathrm{h}} 34^{\mathrm{m}} 15^{\mathrm{s}} .3$ & $22^{\circ} 50^{\prime} 33^{\prime \prime} .1$ & 1.4 & 2.5 \\
\hline 6 & CFHT-BD-Tau 3 & XMM & HP Tau & XEST-08-080 & $04^{\mathrm{h}} 36^{\mathrm{m}} 38^{\mathrm{s}} .9$ & $22^{\circ} 58^{\prime} 13^{\prime \prime} .2$ & 1.9 & 1.2 \\
\hline 7 & CFHT-Tau 6 & $\mathrm{XMM}$ & TMC 1A & XEST-05-005 & $04^{\mathrm{h}} 39^{\mathrm{m}} 04^{\mathrm{s}} .1$ & $25^{\circ} 44^{\prime} 26^{\prime \prime} 4$ & 1.9 & 1.9 \\
\hline 8 & CFHT-BD-Tau 4 & $\mathrm{CXO}$ & L1527 & CXOU J043947.5+260140 & $04^{\mathrm{h}} 39^{\mathrm{m}} 47^{\mathrm{s}} .5$ & $26^{\circ} 01^{\prime} 40^{\prime} 8$ & 0.3 & 0.0 \\
\hline 9 & 2MASS J0455 & XMM & SU Aur & XEST-26-012 & $04^{\mathrm{h}} 55^{\mathrm{m}} 23^{\mathrm{s}} .1$ & $30^{\circ} 27^{\prime} 38^{\prime \prime} .2$ & 2.0 & 3.1 \\
\hline
\end{tabular}

Table 3. Spectral properties of BDs obtained from spectral fitting. To fit the spectra (Fig. 4), we used a WABS absorption model (Morrison \& McCammon 1983) combined with one or two MEKAL optically thin thermal plasma model (Mewe et al. 1995) with 0.3 times the solar elemental abundances. MEKAL plasma models were computed rather than interpolated from a pre-calculated table. We used $\chi^{2}$ statistics with standard weighting. Column (4) gives the net source counts collected by the instrument given in Col. (3). Confidence ranges at the $68 \%$ level $\left(\Delta \chi^{2}=1\right.$; corresponding to $\sigma=1$ for Gaussian statistics) are given in parentheses. The value of reduced $\chi^{2}$ and $v$, the degrees of freedom, are indicated in Col. (10). The emission measures in Cols. (8) and (9) and the X-ray luminosity in the $0.5-8 \mathrm{keV}$ energy range corrected for absorption in Col. (11) were computed assuming a distance of $140 \mathrm{pc}$ for the TMC. The X-ray fractional luminosity, $\eta=\log \left(L_{\mathrm{X}} / L_{*}\right)$, is given in the last column. For CFHT-BD-Tau 1, the second line gives an estimate of the quiescent X-ray luminosity derived for the light curve fit (see Sect. 3).

\begin{tabular}{|c|c|c|c|c|c|c|c|c|c|c|c|}
\hline \# & BD name & Instr. & 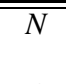 & $\begin{array}{c}N_{\mathrm{H}} \\
10^{21} \mathrm{~cm}^{-2}\end{array}$ & $\overline{\bar{k} k T_{1}}$ & $\overline{k T_{2}}$ & $\begin{array}{r}E M_{1} \\
10^{5}\end{array}$ & $\begin{array}{l}E M_{2} \\
\mathrm{~m}^{-3}\end{array}$ & $\overline{\chi_{\nu}^{2}(v)}$ & $\begin{array}{c}L_{\mathrm{X}} \\
10^{28} \mathrm{erg} \mathrm{s}^{-1}\end{array}$ & $\eta$ \\
\hline (1) & (2) & (3) & (4) & (5) & (6) & (7) & (8) & (9) & (10) & (11) & (12) \\
\hline$\overline{1}$ & 2MASS J0422 & pn & 102 & $1.7(0.9-2.8)$ & $0.7(0.6-0.8)$ & & 2 & & $0.83(07)$ & 02.0 & -3.5 \\
\hline $3 a$ & MHO 4 & EPIC & 571 & $1.2(0.7-1.7)$ & $0.4(0.3-0.5)$ & $1.1(1.0-1.2)$ & 5 & 6 & $0.56(26)$ & 08.3 & -3.3 \\
\hline $3 b$ & MHO 4 & ACIS-I & 271 & $0(0-0.2)$ & $0.5(0.4-0.6)$ & $1.1(1.1-1.3)$ & 4 & 6 & $0.85(18)$ & 08.0 & -3.4 \\
\hline $4 a$ & CFHT-Tau 5 & EPIC & 314 & $4.9(3.7-7.7)$ & $1.5(1.2-1.9)$ & $\ldots$ & 14 & $\ldots$ & $0.82(13)$ & 11.8 & -3.4 \\
\hline 5 & CFHT-BD-Tau 1 & EPIC & 206 & $6.0(3.9-10.2)$ & $1.7(1.1-2.6)$ & & 17 & .. & $0.59(06)$ & $\begin{array}{l}14.3 \\
07.7\end{array}$ & $\begin{array}{l}-2.7 \\
-2.9\end{array}$ \\
\hline 8 & CFHT-BD-Tau 4 & ACIS-I & 33 & $8.6(5.6-13.9)$ & $0.5(0.2-0.9)$ & & 27 & $\ldots$ & $0.74(3)$ & 24.3 & -3.0 \\
\hline
\end{tabular}

Table 4. Spectral properties of BDs obtained from quantile analysis. Column (3) gives the instrument name (MOS stands for MOS1+MOS2). Column (4) and (5) indicate the exposure and the net source counts collected by this instrument (i.e. for MOS the exposure average and the sum of net source counts). Column (6) gives the energies below which the net counts are $25 \%, 50 \%$, and $75 \%$ of the source net counts in the $0.5-7.3 \mathrm{keV}$ energy range. The resulting position in the quantile diagram (Fig. 5) is given in Col. (7). The observed optical extinctions (see Table 1) were used to disentangle temperature double solutions in the quantile diagram (\#2 on pn), and to estimate the hydrogen column density (Col. 8) for sources with low constraint in the quantile diagram (\#2, \#6 and \#7 on pn, and \#9 on MOS), using the relation $N_{\mathrm{H}}=1.6 \times 10^{21} A_{\mathrm{V}} \mathrm{cm}^{-2} \mathrm{mag}^{-1}$ (Vuong et al. 2003; Cardelli et al. 1989); these values are between brackets in Col. 8. Where negligible optical extinction was measured, we adopted $N_{\mathrm{H}}=0.1 \times 10^{21} \mathrm{~cm}^{-2}$. When no constraint on the plasma temperature was obtained, we adopted $1 \mathrm{keV}$ (value between brackets in Col. 9). The $\mathrm{X}$-ray luminosity in the $0.5-8 \mathrm{keV}$ energy range corrected for absorption in Col. (10) was computed assuming a distance of $140 \mathrm{pc}$ for the TMC. The X-ray fractional luminosity, $\eta=\log \left(L_{\mathrm{X}} / L_{*}\right)$, is given in the last column.

\begin{tabular}{|c|c|c|c|c|c|c|c|c|c|c|}
\hline $\begin{array}{c}\# \\
(1)\end{array}$ & $\overline{\text { BD name }}$ & Instr. & $\begin{array}{c}\text { Exp. } \\
\text { ks } \\
(4)\end{array}$ & (5) & $\begin{array}{c}E_{25 \%} / E_{50 \%} / E_{75 \%} \\
\mathrm{keV} \\
(6)\end{array}$ & $\begin{array}{l}\overline{(x, y)} \\
(7)\end{array}$ & $\begin{array}{c}N_{\mathrm{H}} \\
10^{21} \mathrm{~cm}^{-2} \\
(8)\end{array}$ & $\begin{array}{l}k T \\
\mathrm{keV} \\
(9)\end{array}$ & $\begin{array}{c}L_{\mathrm{X}} \\
10^{28} \mathrm{erg} \mathrm{s}^{-1} \\
(10)\end{array}$ & (11) \\
\hline 2 & KPNO-Tau 5 & $\mathrm{pn}$ & 17.2 & 25.3 & $0.71 / 0.82 / 1.02$ & $(-1.31,1.21)$ & [0.1] & 0.2 & 0.8 & -4.1 \\
\hline $4 a$ & CFHT-Tau 5 & pn & 26.1 & 118.4 & $1.08 / 1.45 / 1.89$ & $(-0.79,1.25)$ & 6.4 & 1.4 & 13.9 & -3.3 \\
\hline $4 \mathrm{~b}$ & CFHT-Tau 5 & MOS & 22.9 & 26.6 & $1.02 / 1.27 / 1.60$ & $(-0.90,1.42)$ & 11.1 & 0.5 & 20.1 & -3.2 \\
\hline 5 & CFHT-BD-Tau 1 & pn & 20.6 & 64.7 & $1.11 / 1.36 / 1.98$ & $(-0.84,1.24)$ & 4.5 & 1.5 & 12.4 & -2.7 \\
\hline 6 & CFHT-BD-Tau 3 & pn & 23.9 & 11.6 & $0.79 / 0.98 / 1.19$ & $(-1.12,1.27)$ & [0.1] & [1] & 0.6 & -3.7 \\
\hline 7 & CFHT-Tau 6 & pn & 14.2 & 31.8 & $0.92 / 0.98 / 1.26$ & $(-1.13,1.65)$ & [0.7] & 0.1 & 2.8 & -3.5 \\
\hline 9 & 2MASS J0455 & MOS & 123.9 & 5.0 & $0.75 / 0.95 / 1.15$ & $(-1.15,1.14)$ & {$[0.1]$} & [1] & 0.2 & -4.5 \\
\hline
\end{tabular}



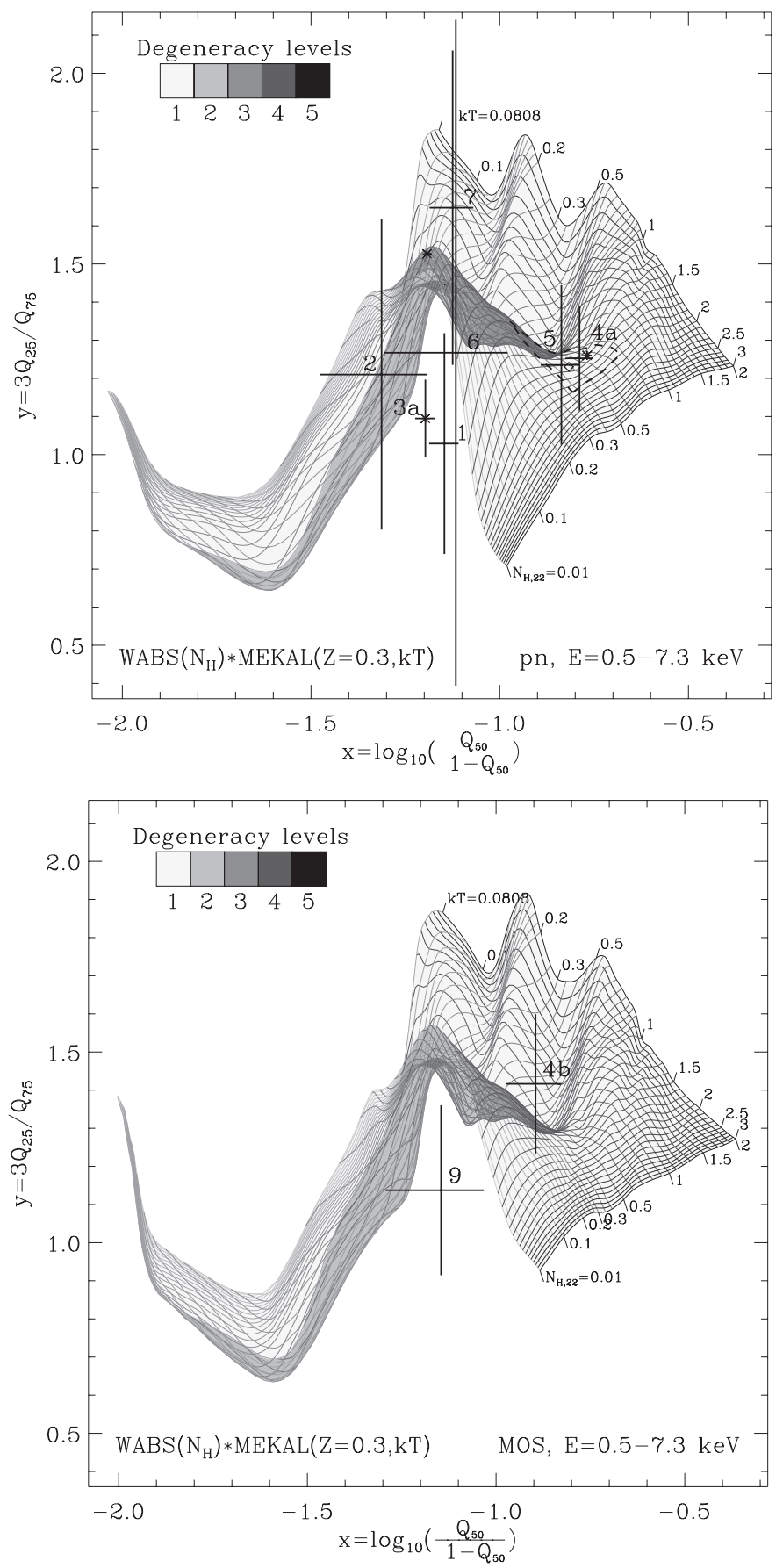

Fig. 5. Quantile diagram for pn (top) and MOS1+MOS2 (bottom). The grid of hydrogen column density and plasma temperature were computed following the prescriptions of Hong et al. (2004) using in SHERPA an absorption model (WABS; Morrison \& McCammon 1983) multiplied with a single optically thin thermal plasma model (MEKAL; Mewe et al. $1995)$ with 0.3 times the solar elemental abundances. The background map in grey levels indicates the degeneracy level of grid parameters (see online Appendix C). The MOS1+MOS2 quantile diagram is used only for X-ray sources without available pn data. In the PN quantile diagram, asterisks and diamond mark the plasma parameters obtained from spectral fitting; the dashed line indicates the X-ray colour locus corresponding to the parameter uncertainties of source \#4a (diamond; Table 3 ). The spectrum of \#3 requires a two-temperature plasma model (see Table 3).

temperatures $\left(N_{\mathrm{H}}, k T\right)$ in SHERPA ${ }^{4}$ and the corresponding $\mathrm{X}$-ray colours. We adopted on-axis RMF and ARF; and for

\footnotetext{
${ }^{4}$ SHERPA is a part of the CIAO package.
}

MOS1+MOS2, the MOS1 and MOS2 RMFs were averaged and the ARFs added. Figure 5 shows the resulting quantile diagrams. Grid lines indicate loci of iso-column densities and isotemperatures.

We noted that the emission lines of the spectral model produce folds in the parameter grid, introducing some degeneracy of the X-ray colours; i.e. several different values of $\left(N_{\mathrm{H}}, k T\right)$ have the same $E_{25 \%}, E_{50 \%}, E_{75 \%}$, and hence the same X-ray colours. The degeneracy of the quantile diagram is studied in details in online Appendix C. The result of this analysis is the degeneracy map plotted in grey levels in Fig. 5, which indicates the number of different $\left(N_{\mathrm{H}}, k T\right)$ values at each position in the quantile diagram.

We calculated for all XMM-Newton sources pn (or MOS1+MOS2) X-ray colours with background subtraction (Table 4) and errors following the prescriptions of Hong et al. (2004). The MOS1+MOS2 quantile diagram is used only for X-ray sources without available pn data. We checked for sources \#1, \#4a and \#5 that the plasma parameters derived from the pn X-ray colours are consistent with the plasma parameters obtained from spectral fitting marked with asterisks and diamond in Fig. 5. For comparison, we indicate in Fig. 5 the X-ray colour locus corresponding to the parameter uncertainties found in the fitting of the XMM-Newton/EPIC spectra of source \#4a, the brightest X-ray source (Table 3). The spectrum of source \#3 requires to be fitted by a two-temperature plasma model, and has X-ray colours which are outside the grid computed with a single-temperature plasma model. Optical extinctions were used to disentangle temperature double solutions (source \#2 on pn; see online Appendix C), and to estimate the hydrogen column density for sources with low constrained X-ray colours (sources \#2, \#6 and \#7 on pn, and \#9 on MOS) using the relation $N_{\mathrm{H}}=1.6 \times 10^{21} A_{\mathrm{V}} \mathrm{cm}^{-2} \mathrm{mag}^{-1}$ (Vuong et al. 2003; Cardelli et al. 1989). Where negligible optical extinction was measured, we adopted $N_{\mathrm{H}}=0.1 \times 10^{21} \mathrm{~cm}^{-2}$. When there was no constraint on the plasma temperature, we adopted $k T=1 \mathrm{keV}$ (Table 4).

Then, in XSPEC we computed from these plasma parameters and the source's ARF and RMF the X-ray luminosity, corrected for absorption in the $0.5-8 \mathrm{keV}$ energy range needed to reproduce the observed net count rate in the $0.5-7.3 \mathrm{keV}$ energy range. For comparison, the X-ray luminosities obtained by spectral fitting of sources \#5a and \#6 and the ones obtained by quantile analysis show only a difference of $\sim 0.1$ dex.

In the following, for BDs observed and detected twice, we use the logarithmic average of their X-ray luminosity. For CFHT-BD-Tau 1, we use the quiescent X-ray luminosity.

\subsection{Upper limit estimate of the $X$-ray luminosities for the undetected brown dwarfs}

We calculated an upper limit to the X-ray luminosity of each undetected BD in the $0.5-8 \mathrm{keV}$ band (see Table 5). Counts within $10^{\prime \prime}$ of the optical/near infrared position were extracted from the summed EPIC soft-band $(0.5-2 \mathrm{keV})$ image. The expected number of counts in the absence of emission from the BD was determined from the identical region of the corresponding "reconstructed image", which is composed of background and detected sources output by the XMM-Newton Science Analysing System (SAS) source detection algorithm, EMLDETECT (Güdel et al. 2007). An upper limit, at the 95\% confidence level, to the number of counts in the region from the BD was computed using the prescription of Kraft et al. (1991), which accounts for Poissonian fluctuations in the counts from the background, and 
those from the $\mathrm{BD}^{5}$. This results in an upper limit to the sum of counts collected by three different detectors. The expected number of counts collected by detector $i, N_{i}$, from a source of flux $f_{\mathrm{X}}$ is dependent on the fraction of the total source counts that were collected within the extraction region, $\epsilon_{i}$, the exposure time, $t_{\text {exp, } i}$, and the source energy flux required to produce a count rate of 1 count s $^{-1}, K_{i}$, such that $N_{i}=\epsilon_{i} \times t_{\exp , i} \times f_{\mathrm{X}} / K_{i}$. Therefore, the upper limit to the source energy flux is computed from the upper limit to the sum of source counts in all the detectors, $N=\sum_{i} N_{i}$, as $f_{\mathrm{X}}=N / \sum_{i} \epsilon_{i} \times t_{\mathrm{exp}, i} / K_{i}$. A model source, constructed at the position of the BD from the calibration point spread function used by EMLDETECT, was used to calculate $\epsilon_{i}$, and convolved with exposure images generated by the SAS command EEXPMAP to compute the effective on-axis exposure time at the BD position, $t_{\exp , i}$, on each detector. The on-axis response of each detector was generated using the SAS command ARFGEN and the appropriate canned response matrix. $K_{i}$ was calculated in XSPEC assuming an isothermal coronal plasma of temperature $1 \mathrm{keV}(11.6 \mathrm{MK})$ with metallicity 0.3 times that of the solar corona (Anders \& Grevesse 1989), and a hydrogen column density obtained from the optical extinction using the relation $N_{\mathrm{H}}=1.6 \times 10^{21} A_{\mathrm{V}} \mathrm{cm}^{-2} \mathrm{mag}^{-1}$ (Vuong et al. 2003; Cardelli et al. 1989). Upper limits to X-ray luminosities in the $0.5-8 \mathrm{keV}$ energy range were calculated assuming a distance of $140 \mathrm{pc}$ to the TMC.

The median of the upper limits of our sample is about $5 \times$ $10^{27} \mathrm{erg} \mathrm{s}^{-1}$. However, the deep archival observation of SU Aur helped to detect 2MASS J0455 down to half this luminosity level.

\section{X-ray luminosities of the XEST brown dwarfs compared to the XEST low-mass stars}

To put the X-ray luminosity properties of the XEST BDs into context, we compare them to the X-ray luminosity of low-mass stars in the XEST (see Güdel et al. 2007). For objects observed and detected twice, we use the logarithmic average of their $\mathrm{X}$-ray luminosity. For TMC members that are unresolved multiple stars in X-rays, we considered the total bolometric luminosities. Figure 6 shows, for the XEST low-mass stars $\left(L_{*} \leq\right.$ $10 L_{\odot}$, which corresponds at 3 Myr to $M \leq 2 M_{\odot}$; Siess et al. 2000), protostars (Class I sources) and BDs, the X-ray luminosities ${ }^{6}$ compared to the bolometric luminosities. Considering only the X-ray detected BDs and low-mass (proto)stars, we determined with the parametric EM (Expectation-Maximization) algorithm implemented in ASURV (Feigelson \& Nelson 1985) the following linear regression fit: $\log \left(L_{\mathrm{X}} / \mathrm{erg} \mathrm{s}^{-1}\right)=(30.06 \pm$ $0.05)+(0.98 \pm 0.06) \times \log \left(L_{*} / L_{\odot}\right)$, with a standard deviation of 0.4 dex in $\log \left(L_{\mathrm{X}}\right)$ for the low-luminosity objects $\left(L_{*} \leq 10 L_{\odot}\right)$.

5 The BD 2MASS J0414 is located on the highly structured PSF wings of the bright X-ray source V773 Tau, which are not wellmodeled, and consequently the contribution of V773 Tau in the extraction region of this $\mathrm{BD}$ is not well estimated. Therefore, we assumed that the number of counts observed in the BD extraction region $\left(6^{\prime \prime}\right.$-radius here instead of $10^{\prime \prime}$-radius) contains a negligible number of counts from the $\mathrm{BD}$, and so the number of observed counts is identical to the number expected from background alone.

${ }^{6}$ For the low-mass (proto)stars, we use the X-ray luminosities in the $0.3-10 \mathrm{keV}$ energy band derived from the DEM method in Güdel et al. (2007). For a BD plasma with 0.3 times the solar elemental abundances and a typical temperature of $1 \mathrm{keV}$, the difference of $\mathrm{X}$-ray luminosity in the $0.3-10 \mathrm{keV}$ energy band and in the $0.5-8 \mathrm{keV}$ energy band is only $0.06 \mathrm{dex}$, and hence can be neglected.
Table 5. Upper limits to the BD X-ray luminosities. The hydrogen column density in unit of $10^{21} \mathrm{~cm}^{-2}$ given in Col. (3) is obtained from the optical extinction using the relation $N_{\mathrm{H}, 21}=1.6 \times A_{\mathrm{V}} \mathrm{cm}^{-2} \mathrm{mag}^{-1}$ (Vuong et al. 2003; Cardelli et al. 1989). Exposure times in Col. (4) are for summed EPIC (pn+M1+M2) data, in units equivalent for a pn-onaxis observation. Columns (5) and (6) give the upper limits at the $95 \%$ confidence level for net counts in the $0.5-2 \mathrm{keV}$ energy range and the $\mathrm{X}$-ray luminosity in the $0.5-8 \mathrm{keV}$ energy range corrected for absorption, respectively. The upper limit to the X-ray fractional luminosity, $\eta=\log \left(L_{\mathrm{X}} / L_{*}\right)$, is given in the last column.

\begin{tabular}{|c|c|c|c|c|c|c|}
\hline BD name & $\begin{array}{c}\text { XEST } \\
\# \\
(2)\end{array}$ & $\begin{array}{c}N_{\mathrm{H}, 21} \\
\mathrm{~cm}^{-2} \\
(3)\end{array}$ & $\begin{array}{c}\text { Exp. } \\
\text { ks } \\
(4)\end{array}$ & $\begin{array}{c} \\
\text { cnts } \\
(5)\end{array}$ & $\begin{array}{c}L_{\mathrm{X}, 27} \\
\mathrm{erg} \mathrm{s}^{-1} \\
(6)\end{array}$ & (7) \\
\hline $2 \mathrm{MASS}$ J0414 & 20 & 1.1 & 44.7 & 115.5 & 15.8 & -3.6 \\
\hline $\mathrm{M}$ & 11 & 4.8 & 38. & 19 & 6. & -3.3 \\
\hline $\mathrm{PN}$ & $23+24$ & 0.6 & 22.1 & 30.2 & 6. & -3.6 \\
\hline PNO-Tau 4 & 02 & 3.9 & 51.8 & 25.8 & 5.0 & -3.5 \\
\hline KPNO-Tau 6 & 14 & 0.1 & 26.8 & 31.1 & 6.4 & -3.3 \\
\hline PNO-Tau 7 & 14 & 0.1 & 25.6 & 16.9 & 2.5 & -3.8 \\
\hline PNO-Tau & $08+09$ & 0.1 & 51.5 & 37.9 & 2.8 & -3.1 \\
\hline CFHT-BD-Tau 2 & 08 & 0.1 & 33.9 & 25.5 & 2.9 & -4.0 \\
\hline
\end{tabular}

This relation is very similar to the relations found for lowmass stars in other young clusters (Feigelson \& Montmerle 1999; Preibisch et al. 2005a). It is consistent with a linear relation between $\mathrm{X}$-ray and bolometric luminosity characterized by $\left\langle\log \left(L_{\mathrm{X}} / L_{*}\right)\right\rangle=-3.5 \pm 0.4$ which is valid from the low-mass stars to the substellar regime. However, the bulk of the upper limits of undetected BDs are below this average X-ray fractional luminosity, which suggests a lower X-ray fractional luminosity for the BDs with about $L_{*} \leq 0.02 L_{\odot}$. Taking into account upper limits of undetected BDs, the median of $\log \left(L_{X} / L_{*}\right)$ for the XEST BDs is -4.0 (see Sect. 7). The X-ray fractional luminosity of XEST BDs is hence lower than the one of XEST low-mass stars.

To investigate the relation between X-ray fractional luminosity and physical parameters when one moves from lowmass stars to the substellar regime, we focus on objects with spectral type M0 or later, which corresponds for an age of $3 \mathrm{Myr}$ to masses and luminosities lower than about $1 M_{\odot}$ and $0.7 L_{\odot}$, respectively. We used the temperature scale given in Table 1 to convert $M$ spectral types to effective temperatures. We determined source masses from effective temperature and luminosities, using the pre-main-sequence tracks of Baraffe et al. (1998). Masses were interpolated linearly along the isochrones. For sources located above the $1 \mathrm{Myr}$ isochrone, we prolonged the convective tracks vertically. We attributed $0.02 M_{\odot}$ to KPNO-Tau 4, i.e. the only BD above the $0.02 M_{\odot}$ track. The source IRAS S04301+261 (spectral type M0), which is located below the main-sequence was discarded. We restricted our sample to stars which are not multiple in order not to introduce extra assumptions when distributing the X-ray flux to unresolved components. Figure 7 shows the X-ray fractional luminosity versus mass for this sample. Spearman's rank correlation coefficient computed with ASURV indicates a correlation with the probability of the null hypothesis (i.e. no correlation) $P(0) \leq 0.06$. The EM algorithm yields the shallow linear regression fit: $\log \left(L_{\mathrm{X}} / L_{\mathrm{bol}}\right)=(-3.5 \pm 0.1)+(0.3 \pm 0.2) \times \log \left(M / M_{\odot}\right)$, with a standard deviation of $0.5 \mathrm{dex}$ in $\log \left(L_{\mathrm{X}} / L_{*}\right)$. This relation is consistent with the relation found by Preibisch et al. (2005a) for the T Tauri stars $\left(M \leq 2 M_{\odot}\right)$ of the ONC. This relation implies that the X-ray fractional luminosity decreases by a factor of about 3 from $1 M_{\odot}$ stars to $0.03 M_{\odot}$ BDs. 


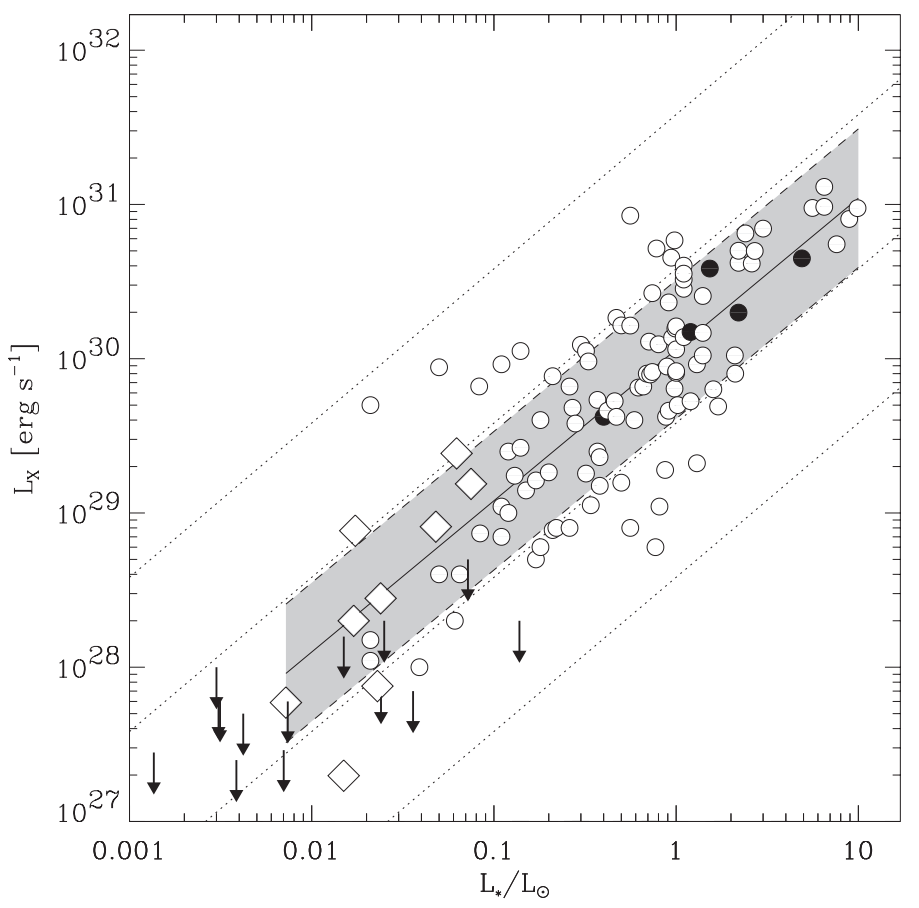

Fig. 6. X-ray luminosity vs. bolometric luminosity for the TMC BDs (diamonds; and arrows for upper limits) and the TMC members detected in the XEST (white and black dots represent low-mass stars and protostars, respectively; Güdel et al. 2007). The dotted lines indicate from bottom to top an X-ray fractional luminosity, $\eta=\log \left(L_{X} / L_{*}\right)$, of $-5,-4,-3,-2$. The grey stripe shows a linear regression fit (continuous line) and standard deviation (dashed lines) for detected objects with bolometric luminosities lower than $10 L_{\odot}$.

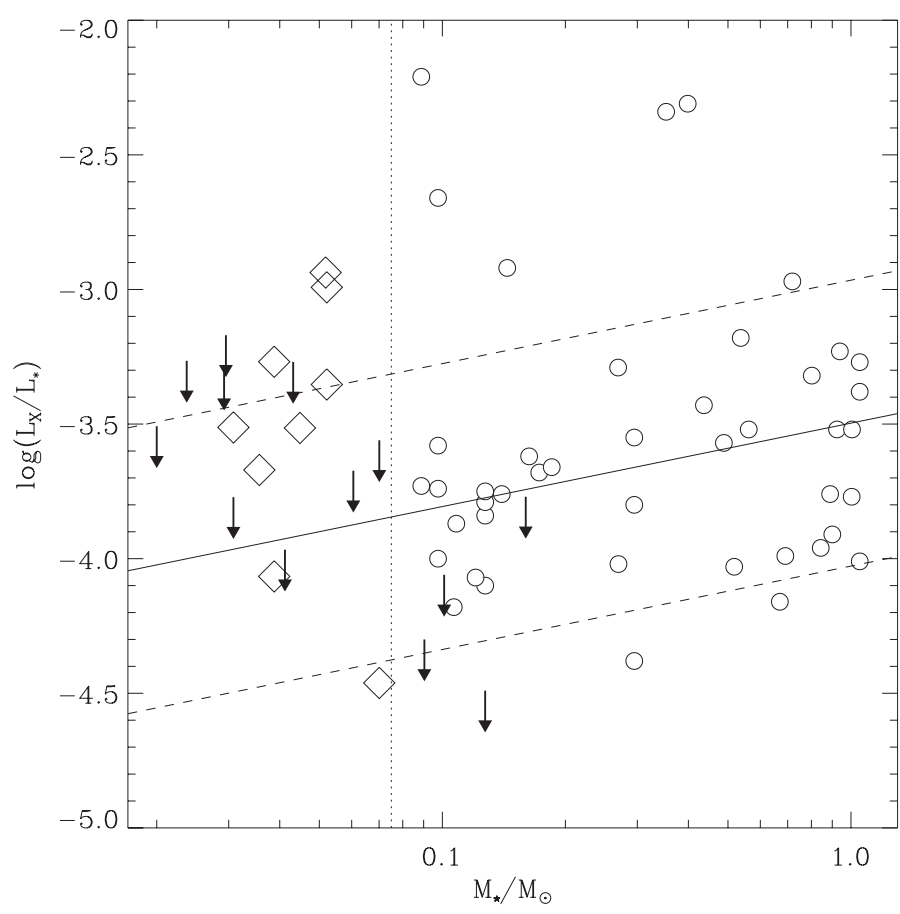

Fig. 7. X-ray fractional luminosity vs. mass for the young BDs of the TMC and single TMC members of the XEST. The symbols are as in Fig. 6. The vertical dotted lines indicates the stellar/substellar boundary. The solid and dashed lines show a linear regression fit and standard deviation, respectively.

Figure 8 shows the X-ray fractional luminosity versus the effective temperature for our sample ranging from $\sim 3840 \mathrm{~K}$

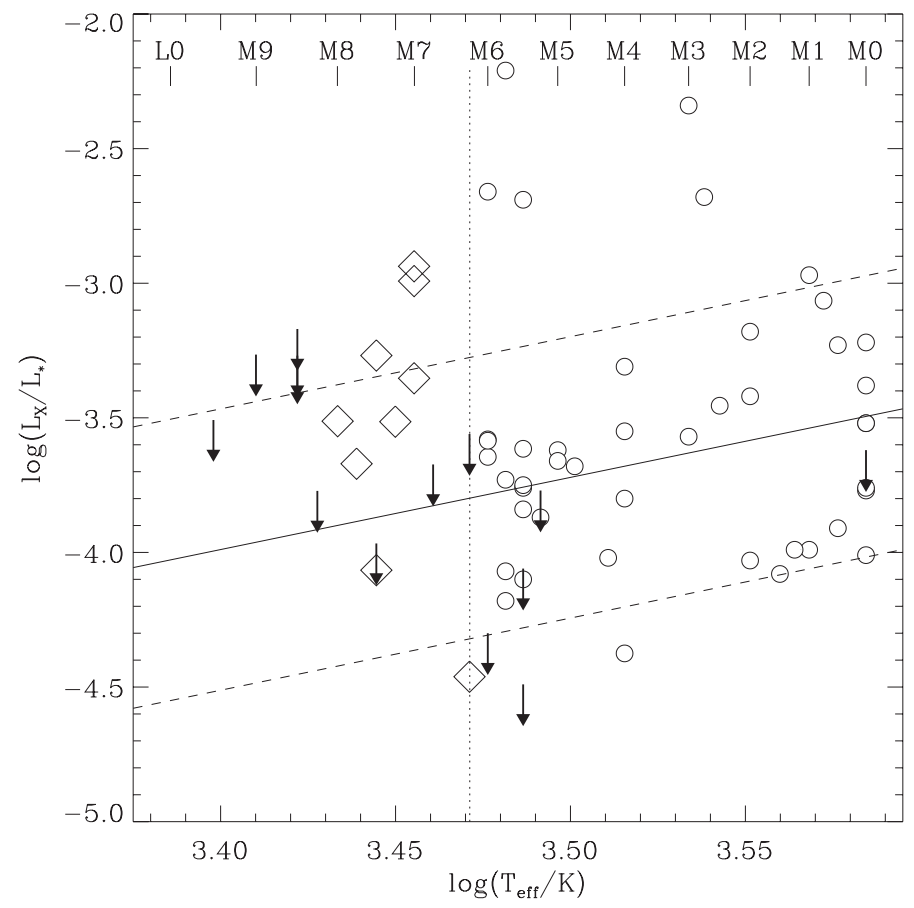

Fig. 8. X-ray fractional luminosity vs. effective temperature for the young BDs of the TMC and single TMC members of the XEST. The symbols are as in Fig. 6 . The vertical dotted lines indicates the stellar/substellar boundary. The solid and dashed lines show a linear regression fit and standard deviation, respectively.

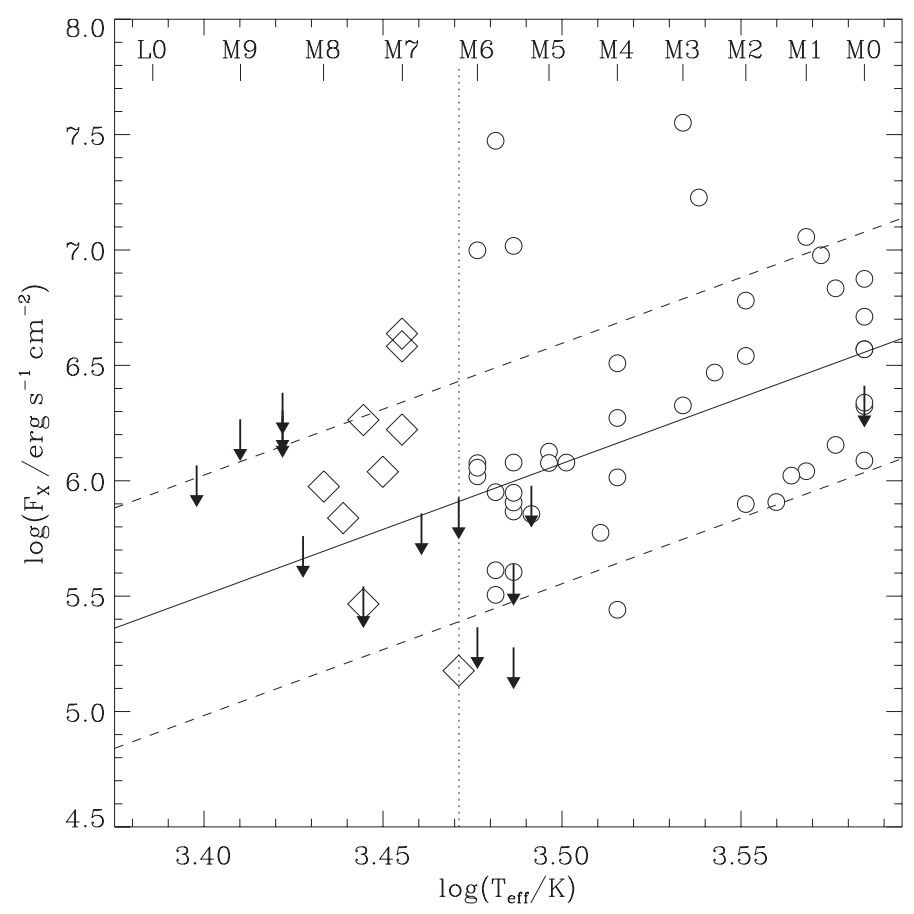

Fig. 9. X-ray surface flux vs. effective temperature for the young BDs of the TMC and single TMC members of the XEST with spectral type M0 or later. The symbols are as in Fig. 6. The solid and dashed lines show a linear regression fit and standard deviation, respectively.

to $2500 \mathrm{~K}$. Spearman's rank correlation coefficient computed with ASURV indicates a correlation with the probability of the null hypothesis (i.e. no correlation) $P(0) \leq 0.11$. The EM algorithm yields the linear regression fit: $\log \left(L_{\mathrm{X}} / L_{\mathrm{bol}}\right)=(-13.1 \pm$ $5.4)+(2.7 \pm 1.5) \times \log \left(T_{\text {eff }} / \mathrm{K}\right)$, with a standard deviation of $0.5 \mathrm{dex}$ 
in $\log \left(L_{\mathrm{X}} / L_{*}\right)$. This relation implies that the X-ray fractional luminosity decreases by a factor of about 3 from hot coronae of solar-mass stars to cooler atmospheres of M9V BDs.

We computed the X-ray surface flux, i.e. the X-ray luminosity divided by the surface area of the source, which is computed from its bolometric luminosity and effective temperature: $F_{\mathrm{X}}=\sigma T_{\text {eff }}^{4} L_{\mathrm{X}} / L_{*}$, where $\sigma$ is the Stefan-Boltzmann constant. Combined with the decrease of the $L_{\mathrm{X}} / L_{*}$ ratio with the effective temperature, this formula implies that the $\mathrm{X}$-ray surface flux decreases with the effective temperature with a power-law slope greater than 4 . Figure 9 shows the X-ray surface flux versus the effective temperature for our sample. The X-ray surface fluxes range from $\sim 10^{5}$ to $\sim 3 \times 10^{7} \mathrm{erg} \mathrm{s}^{-1} \mathrm{~cm}^{-2}$. For comparison, the $\mathrm{X}$-ray surface flux of the solar corona during the solar cycle varies from $\sim 2 \times 10^{3}$ to $\sim 8 \times 10^{4} \mathrm{erg} \mathrm{s}^{-1} \mathrm{~cm}^{-2}$ (Peres et al. 2004). Spearman's rank correlation coefficient indicates a log-log correlation with a high confidence level $\left(P(0)=10^{-4}\right)$. The EM algorithm yields: $\log \left(F_{\mathrm{X}} / \mathrm{erg} \mathrm{s}^{-1} \mathrm{~cm}^{-2}\right)=(-13.9 \pm 4.8)+(5.7 \pm 1.4) \times$ $\log \left(T_{\text {eff }} / \mathrm{K}\right)$, with a standard deviation of 0.5 dex in $\log \left(F_{\mathrm{X}}\right)$. The slope of this correlation is well steeper than 4 due to the decline of the $L_{X} / L_{*}$ ratio with the effective temperature. This correlation predicts that a M9V BD should have an X-ray surface flux in its corona $\sim 10$ times weaker than in an M0 star. However, this is still 4 times higher than the X-ray surface flux of the solar corona at the solar cycle maximum (Peres et al. 2004). A similar trend was found by Preibisch et al. (2005b) for the BDs of ONC (see Sect. 7 for further comparison between the XEST and the COUP BD sample).

\section{X-ray fractional luminosity and $\mathrm{H} \alpha$ emission of the XEST brown dwarfs}

\subsection{Correlation between $X$-rays and $\mathrm{H} \alpha_{\alpha}$ ?}

Studying a BD sample including young BDs (in the ONC, $\rho$ Ophiuchi, and IC 348) plus one intermediate BD (TWA 5B) and one old (LP 944-20) field BD, Tsuboi et al. (2003) found a $\log$-log correlation between the X-ray fractional luminosity (value independent of the distance assumption), and $E W(\mathrm{H} \alpha)$ $\left(\log L_{\mathrm{X}} / L_{*}=-5.3+1.5 \times \log E W(\mathrm{H} \alpha)\right)$, and noted that BDs with $E W(\mathrm{H} \alpha)$ greater than $100 \AA$ were not detected in X-rays. We note that including the corresponding (six) X-ray upper limits, this correlation vanishes. Moreover, this proposed correlation is strongly biased by the low X-ray activity of LP 944-20 - detected only during an X-ray flare (Rutledge et al. 2000) - which is likely explained by the cooling of this old (500 Myr) field BD (Stelzer et al. 2006).

$E W(\mathrm{H} \alpha)$ measurements are available for all the XEST BDs but 2MASS J0455 (Table 1), allowing us to investigate the relationship between X-ray fractional luminosity and $\mathrm{H} \alpha$ emission from a well-defined sample of young BDs. Figure 10 shows the X-ray fractional luminosity versus $E W(\mathrm{H} \alpha)$. We detected in X-rays BDs both with low and high $E W(\mathrm{H} \alpha)$. We compute Spearman's rank correlation coefficient, and find no significant $\log -\log$ correlation between the X-ray fractional luminosity and $E W(\mathrm{H} \alpha)$.

\subsection{X-ray activity and accretion}

The $\mathrm{H} \alpha$ emission line cannot be used as a tracer of accretion in low-mass stars and BDs without a priori knowledge on the limit of pure chromospheric $\mathrm{H} \alpha$ emission in these objects. The canonical limit of $10 \AA$ for $E W(\mathrm{H} \alpha)$ was first used to disentangle pure chromospheric activity in Weak-line T Tauri Stars (WTTSs)

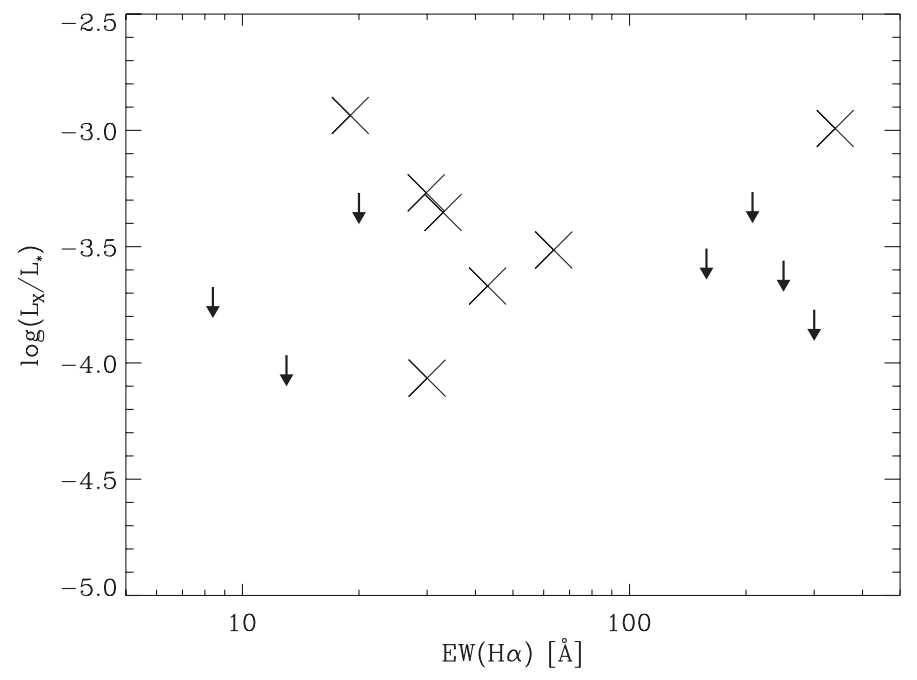

Fig. 10. X-ray fractional luminosity of TMC BDs vs. H $\alpha$. TMC BDs detected in X-rays are marked with "X".

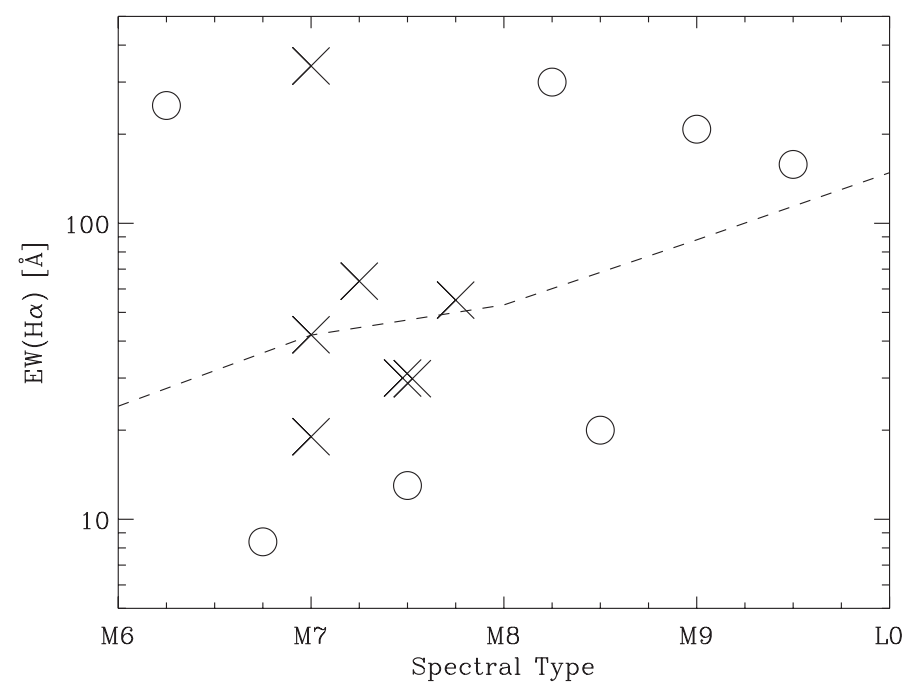

Fig. 11. Equivalent width of $\mathrm{H} \alpha$ emission lines vs. spectral type for TMC BDs. TMC BDs detected in X-rays are marked with " $\mathrm{X}$ ". The dashed line shows the saturation limit of chromospheric activity at $\log \left[L(\mathrm{H} \alpha) / L_{*}\right]=-3.3$ (determined in the open clusters; Barrado y Navascués \& Martín 2003).

and $\mathrm{H} \alpha$ emission line excess in Classical T Tauri stars (CTTSs) produced by accretion. Martín (1998) proposed $E W(\mathrm{H} \alpha)$ limits depending on the spectral type. Barrado y Navascués \& Martín (2003) improved this criterion and extended it into the substellar domain. This empirical criterion is the saturation at $\log \left[L(\mathrm{H} \alpha) / L_{*}\right]=-3.3$, based on observations of nonaccreting stars in young open clusters, which corresponds physically to the maximum amount of energy that can be released in nonthermal processes by the chromosphere, i.e. $\sim 5 \times 10^{-4}$ of the total emitted energy. Low-mass stars or BDs exceeding this limit are accreting. Figure 11 shows $E W(\mathrm{H} \alpha)$ versus spectral type for the BDs of XEST. The dashed line shows the saturation at $\log \left[L(\mathrm{H} \alpha) / L_{*}\right]=-3.3$ which increases from $E W(\mathrm{H} \alpha) \sim 24 \AA$ at M 6 to $\sim 148 \AA$ at L0 (Barrado y Navascués \& Martín 2003). MHO 4 and CFHT-BD-Tau 3 are just above this limit, but highresolution spectrum showed no indication of accretion (Mohanty et al. 2005). Therefore, we classify these BD as nonaccreting. For the other BDs, our classification based on $E W(\mathrm{H} \alpha)$ is in agreement with detailed high-resolution accretion identification 


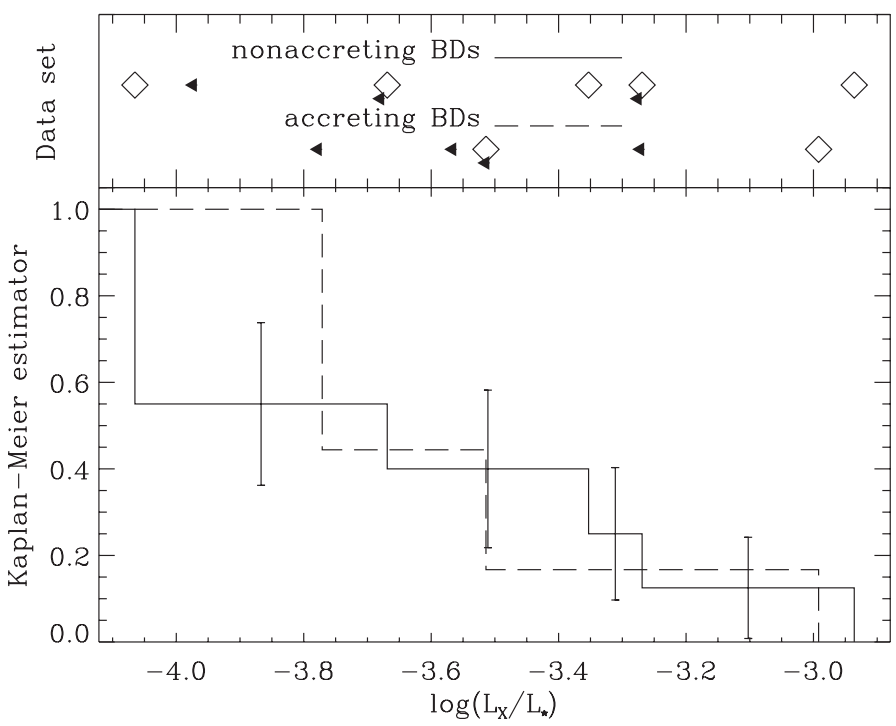

Fig. 12. Cumulative distributions of the $X$-ray fractional luminosities for nonaccreting (continuous line) and accreting (dashed line) BDs. In the top panel, diamonds and arrows indicate detections and upper limits, respectively of the two samples. Only Kaplan-Meier estimator error bars of the nonaccreting sample are shown to clarify the plot. Twopopulation statistical methods show that both samples are drawn from the same underlying distribution.

(Muzerolle et al. 2005b; Mohanty et al. 2005). We find 6 accreting and 8 nonaccreting BDs. The X-ray detection rates of accreting and nonaccreting BDs are 33\% $\pm 27 \%(2 / 6)$ and $63 \% \pm 36 \%$ (5/8), respectively ${ }^{7}$.

We compute with ASURV the cumulative distributions of the X-ray fractional luminosities - including upper limits with the Kaplan-Meier estimator for accreting and nonaccreting BDs (Fig. 12). Two-population statistical methods provided by ASURV $^{8}$ show that the probability for the null hypothesis that both samples are drawn from the same underlying distribution is 0.75 . Therefore, the accreting and nonaccreting BDs have similar X-ray fractional luminosities. The median value of the X-ray fractional luminosities is -3.9 for the nonaccreting BDs.

A large difference is found between accreting and nonaccreting low-mass stars. In the TMC, the CTTSs are 2.2 times less luminous than the WTTS (Güdel et al. 2006). A similar result was found in the ONC where the CTTSs (selected with $8542 \AA$ Ca II line) are 2.1 times less luminous than the WTTSs (Flaccomio et al. 2003; Preibisch et al. 2005a). Preibisch et al. (2005a) proposed that in accreting objects magnetic reconnection cannot heat the dense plasma in mass-loaded accreting field lines to X-ray temperatures. If true, this implies that coronal activity is less affected by the accretion in BDs than in low-mass stars.

The median X-ray fractional luminosity of nonaccreting BDs in the XEST is $\sim 4$ times lower than the mean saturation value for rapidly rotating low-mass $\left(0.22 \leq M_{\star} / M_{\odot} \leq 0.60\right)$ field stars $\left(\log \left(L_{\mathrm{X}} / L_{*}\right)=-3.3\right.$; Pizzolato et al. 2003), whereas in

7 To compute ratio errors we considered that the number of X-ray detected BDs and the number of (non)accreting BDs are not exactly known, and we combined both Poissonian errors using Gaussian propagation, i.e. $\Delta(a / b)=a / b \times \sqrt{(\Delta a / a)^{2}+(\Delta b / b)^{2}}$ with $\Delta a=\sqrt{a}$ and $\Delta b=\sqrt{b}$.

${ }^{8}$ Namely the Gehan and Peto-Peto generalized Wilcoxon tests, and the Logrank test, which are standard methods of univariate survival analysis as described by Feigelson \& Nelson (1985).

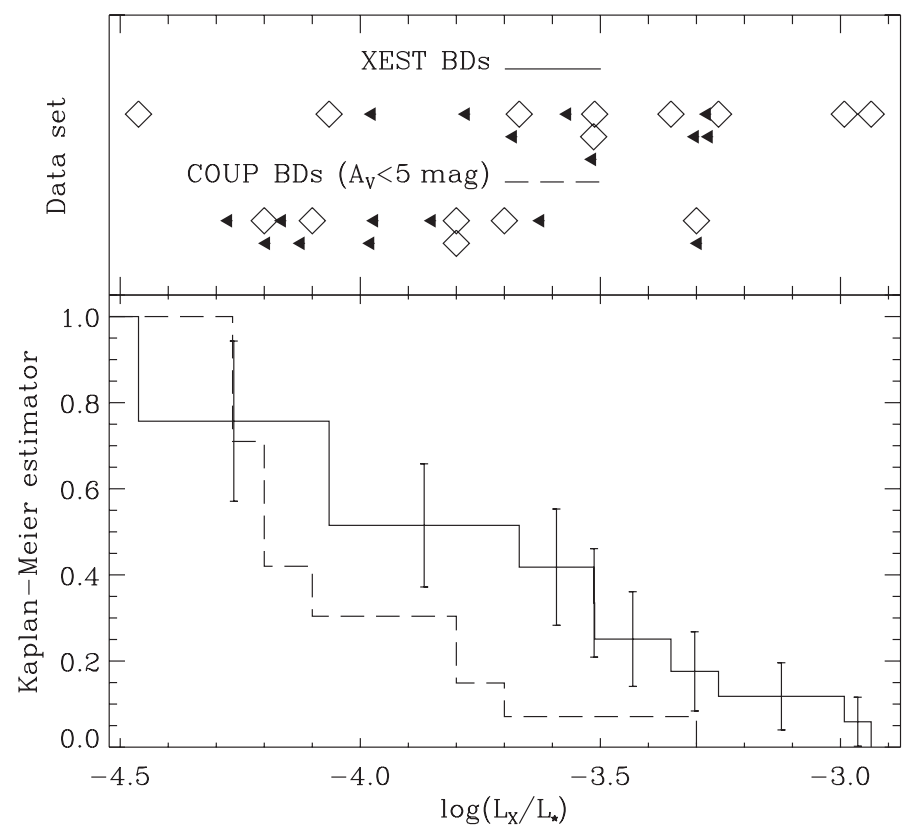

Fig. 13. Cumulative distributions of the $\mathrm{X}$-ray fractional luminosities of XEST (continuous line) and COUP (dashed line) BDs. In the top panel, diamonds and arrows indicate detections and upper limits, respectively of the two samples. Only Kaplan-Meier estimator error bars of the nonaccreting sample are plotted to clarify the plot. Two-population statistical methods show that XEST BDs are more active in X-rays than COUP BDs.

ONC the median X-ray fractional luminosity of nonaccreting low-mass stars is consistent with this saturation level (Preibisch et al. 2005a).

\section{Comparison of brown dwarf X-ray fractional luminosities in the XEST and COUP}

Preibisch et al. (2005b) in COUP studied the X-ray properties of young BDs spectroscopically identified in the near-IR (Slesnick et al. 2004) having spectral types between M6V and M9V. Eight out of the $33 \mathrm{BDs}^{9}$ were clearly detected as X-ray sources down to a detection $\operatorname{limit}$ of $\log \left(L_{\mathrm{X}} / \mathrm{erg} \mathrm{s}^{-1}\right)=27.3$. The near-IR selection helped to find extincted BDs (Slesnick et al. 2004). Consequently, the apparently low detection rate of BDs in COUP is in many cases related to the substantial extinction of these BDs. Considering only the ONC BDs with $A_{\mathrm{V}} \leq 5 \mathrm{mag}$ reduces the median visual extinction from 5.6 to $2.2 \mathrm{mag}$, and leads to an X-ray detection rate of $40 \%$ (6 out of 15 BDs), similar to the X-ray detection rate observed for TMC BDs.

We compute the cumulative distributions of the X-ray fractional luminosities of the whole sample of XEST BDs, and of the COUP BDs with $A_{\mathrm{V}} \leq 5 \mathrm{mag}$ (Fig. 13). Two-population statistical methods show that the probability for the null hypothesis that both samples are drawn from the same underlying distribution is lower than 0.05 . The former and the latter samples have median X-ray fractional luminosity equal to -4.0 and -4.2 , respectively. Therefore, the XEST BDs are 1.6 times more active in X-rays than the COUP BDs. The origin of this difference is not yet understood.

9 We suppressed from the young BD sample of Preibisch et al. (2005b) COUP $344=\mathrm{HC} 722$, which was identified as a foreground (old) dwarf object in Slesnick et al. (2005)'s erratum. This object, detected in COUP only during an X-ray flare, was also discussed in Preibisch et al. (2005b) but kept in the reference sample. 


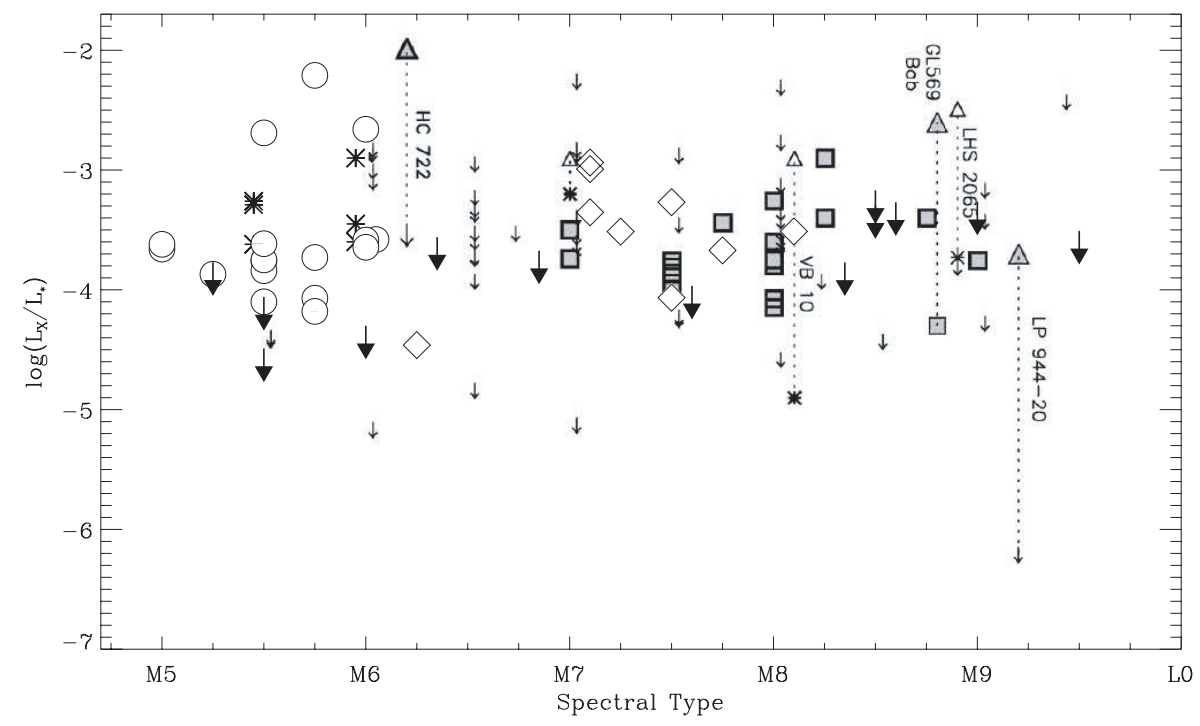

Fig. 14. X-ray fractional luminosity vs. spectral type for objects of type M5 and later. Detections of late $\mathrm{M}$ field stars from Fleming et al. (1993) are shown as asterisks. The circles show low-mass stars of the TMC detected in X-rays (Güdel et al. 2007). Diamonds and thick arrows show BDs in the TMC. The other X-ray detected BDs (see Preibisch et al. 2005b, and references therein) are shown by gray filled squares. For very cool objects with strong flares, the values at flare peak are shown by triangles, connected by dotted lines to the quiescent emission. Some symbols have been slightly moved in spectral type to avoid overlaps.

\section{Discussion}

We discuss now the origin of the BD X-ray emission in the broader context of the X-ray emission of cool stars. Following Preibisch et al. (2005b), we compare the X-ray fractional luminosities of TMC BDs with other cool objects.

Figure 14 shows the X-ray fractional luminosities of TMC objects with spectral type equal or later than M 5, X-ray detected BDs (Preibisch et al. 2005b, and references therein; Stelzer et al. 2006), the evolved BD Gl 569 Ba,b (Stelzer 2004), and the field BD LP 944-20 (Rutledge et al. 2000; Martín \& Bouy 2002). Cool field stars with spectral type M 5 or later are also indicated: M field stars from Fleming et al. (1993); very-low mass field stars VB 10 (Fleming et al. 2003), LHS 2065 (Schmitt \& Liefke 2002), HC 722 (Slesnick et al. 2004, 2005; Preibisch et al. 2005b). All these cool objects show a similar level of X-ray fractional luminosities ranging from $\sim 10^{-3}$ to $\sim 10^{-4}$ (note that, compared to Fig. 8, the spectral type range has been reduced by a factor of two in Fig. 14).

Based on Baraffe et al. (1998) evolutionary tracks, objects of spectral type M 7 with an age of 1 Gyr are not BDs, but lowmass stars twice as massive as a typical TMC BD having an M 7 spectral type and an age of 3 Myr. Moreover, such very cool stars also have surface gravities about 40 times higher than in a typical TMC BD. This shows, as found by Preibisch et al. (2005b) from the COUP BDs, that the X-ray activity of BD coronae is not strongly dependent of the BD mass and the BD surface gravity. Therefore, this implies that the relation that we have obtained, for the young objects of TMC with spectral type $\mathrm{M}$, between X-ray fractional luminosity and mass (Fig. 7) is in fact the consequence of the more fundamental relation between $\mathrm{X}$-ray fractional luminosity and effective temperature (Fig. 8). This latter relation agrees with the overall result (field dwarfs and young BDs) in Fig. 14: of the 15 sources shown with spectral types M8.5V or later, only 4 have any detected quiescent emission; the rest are either not detected at all or (in 3 cases) detected only during strong flares. By considering a subsample of evolved BDs (from $\sim 1 \mathrm{Myr}$ to $\sim 1 \mathrm{Gyr}$ ) with a small range of masses $\left(0.05-0.07 M_{\odot}\right)$ but with effective temperatures ranging from $3000 \mathrm{~K}$ down to $1000 \mathrm{~K}$, Stelzer et al. (2006) found evidence for a similar decline of the fractional X-ray luminosity with the effective temperature; showing that the atmospheric temperature plays a crucial role in determining the level of X-ray activity.

The growing evidence for a decline of the coronal activity with the effective temperature is analogous to the decline in chromospheric $(\mathrm{H} \alpha)$ activity seen in field dwarfs at similar spectral types, which show a slow decline from mid- to late $\mathrm{M}$ in $L_{\mathrm{H} \alpha} / L_{*}$ and a sharp drop-off around M9V (Mohanty \& Basri 2003). Although a definitive turn-over of the fractional X-ray luminosity toward L-type BDs is not proven for the present sample due to sparse statistics, we note that the fractional luminosity declines from low-mass stars to M-type BDs, and as a sample, the BDs are less efficient X-ray emitters than low-mass stars. We thus conclude that while the BD atmospheres observed here are mostly warm enough to sustain coronal activity, a trend is seen that may indicate its gradual decline due to the drop in photospheric ionization degree (Mohanty \& Basri 2003).

\section{Summary}

With the XEST we detected 9 (out of 17) young BDs with spectral type ranging from $\mathrm{M} 6.25 \mathrm{~V}$ to $\mathrm{M} 8 \mathrm{~V} ; 7 \mathrm{BDs}$ are detected here for the first time in X-rays. This BD sample surveyed in X-rays allowed us to investigate the magnetic activity in the substellar regime. We recovered a well-known relation between X-ray and bolometric luminosity for stars (with $L_{*} \leq 10 L_{\odot}$ ) and BDs detected in X-rays, which is consistent with a mean X-ray fractional luminosity $\left\langle\log \left(L_{\mathrm{X}} / L_{*}\right)\right\rangle=-3.5 \pm 0.4$. For the XEST BDs, the median of $\log \left(L_{X} / L_{*}\right)$ (including upper limits) is -4.0 . The $\mathrm{X}$-ray fractional luminosity of XEST BDs is hence lower than the one of XEST low-mass stars.

A shallow relation is found between $\mathrm{X}$-ray fractional luminosity and mass. We show that the X-ray fractional luminosity decline by a factor of about 3 from hot coronae of solar-mass stars to cooler atmospheres of M9V BDs. Consequently, a relation is found between X-ray surface flux and effective temperature, which implies a decrease of about one magnitude in the X-ray surface flux from a M0V star to a M9V BD.

No significant relation is found between the X-ray fractional luminosity and $E W(\mathrm{H} \alpha)$. We used $E W(\mathrm{H} \alpha)$ to identify accreting and nonaccreting BDs. Accreting and nonaccreting BDs have a similar X-ray fractional luminosities. The median X-ray fractional luminosity of nonaccreting BDs are about 4 times lower 
than the mean saturation value for rapidly rotating low-mass field stars. BDs have higher X-ray fractional luminosities in the TMC than in Orion.

We confirm, as previously observed in the Cha I (Stelzer et al. 2004) and in the ONC (Preibisch et al. 2005b), that there is no dramatic change of the magnetic activity at the stellar/substellar boundary. Young BDs of spectral type M are sufficiently warm to sustain an active corona. The young BDs in the TMC, with a median spectral type of M7.5, have on average an $\mathrm{X}$-ray surface flux which is 7 times higher than the one observed in the solar corona at the solar cycle maximum.

Deeper X-ray observations of the coolest M-type BDs in the TMC are needed to investigate a possible turn-over of the fractional X-ray luminosity of TMC BDs around spectral type M9V.

Note added in proof 2MASS J04335245+2612548, a TMC BD with spectral type M8.5V (Luhman et al. 2006) which was reported after the acceptance of this paper, is neither detected in X-rays by $X M M$-Newton/EPIC or in the $U$-band by the XMM-Newton optical/UV monitor; it is located on the X-ray PSF wings and the $U$-band "smoke ring" of IT Tau (XEST-18-030). Therefore, adding this newly identified BD to our TMC BD sample wouldn't change our conclusions.

Acknowledgements. We thank the anonymous referee for his constructive comments that helped to improve this paper; Beate Stelzer for a careful reading of the manuscript; and the International Space Science Institute (ISSI) in Bern for significant financial support of the project team. This research is based on observations obtained with XMM-Newton, an ESA science mission with instruments and contributions directly funded by ESA Member States and NASA. X-ray astronomy research at PSI has been supported by the Swiss National Science Foundation (grants 20-66875.01 and 20-109255/1). M.A. acknowledges support from NASA grant NNG05GF92G.

\section{References}

Anders, E., \& Grevesse, N. 1989, Geochim. Cosmochim. Acta, 53, 197

Arnaud, K. A. 1996, in Astronomical Data Analysis Software and Systems V, ed. G. H. Jacoby, \& J. Barnes, ASP Conf. Ser., 101, 17

Bally, J., Feigelson, E., \& Reipurth, B. 2003, ApJ, 584, 843

Baraffe, I., Chabrier, G., Allard, F., \& Hauschildt, P. H. 1998, A\&A, 337, 403

Baraffe, I., Chabrier, G., Allard, F., \& Hauschildt, P. H. 2002, A\&A, 382, 563

Barrado y Navascués, D., \& Martín, E. L. 2003, AJ, 126, 2997

Briceño, C., Hartmann, L., Stauffer, J., \& Martín, E. 1998, AJ, 115, 2074

Briceño, C., Calvet, N., Kenyon, S., \& Hartmann, L. 1999, AJ, 118, 1354

Briceño, C., Luhman, K. L., Hartmann, L., Stauffer, J. R., \& Kirkpatrick, J. D. 2002, ApJ, 580, 317

Briggs, K. R., \& Pye, J. P. 2004, MNRAS, 353, 673

Cardelli, J. A., Clayton, G. C., \& Mathis, J. S. 1989, ApJ, 345, 245

Carkner, L., Feigelson, E. D., Koyama, K., Montmerle, T., \& Reid, I. N. 1996, ApJ, 464, 286

Comerón, F., Neuhäuser, R., \& Kaas, A. A. 2000, A\&A, 359, 269

Dame, T. M., Ungerechts, H., Cohen, R. S., et al. 1987, ApJ, 322, 706

Dobashi, K., Uehara, H., Kandori, R., et al. 2005, PASJ, 57, 1

Favata, F., Flaccomio, E., Reale, F., et al. 2005, ApJS, 160, 469

Favata, F., Giardino, G., Micela, G., Sciortino, S., \& Damiani, F. 2003, A\&A, 403, 187

Feigelson, E. D., \& Montmerle, T. 1999, ARA\&A, 37, 363

Feigelson, E. D., \& Nelson, P. I. 1985, ApJ, 293, 192

Flaccomio, E., Damiani, F., Micela, G., et al. 2003, ApJ, 582, 398

Flaccomio, E., Micela, G., Sciortino, S., et al. 2005, ApJS, 160, 450

Fleming, T. A., Giampapa, M. S., \& Garza, D. 2003, ApJ, 594, 982

Fleming, T. A., Giampapa, M. S., Schmitt, J. H. M. M., \& Bookbinder, J. A. 1993, ApJ, 410, 387
Garmire, G. P., Bautz, M. W., Ford, P. G., Nousek, J. A., \& Ricker, G. R. 2003 , in X-Ray and Gamma-Ray Telescopes and Instruments for Astronomy, J. E. Truemper, H. D. Tananbaum (ed.), Proc. SPIE, 4851, 28

Getman, K. V., Flaccomio, E., Broos, P. S., et al. 2005, ApJS, 160, 319

Grosso, N., Audard, M., Bouvier, J., Briggs, K., \& Güdel, M. 2007, A\&A, 468, 557

Güdel, M., Audard, M., Kashyap, V. L., Drake, J. J., \& Guinan, E. F. 2003, ApJ, 582,423

Güdel, M., Skinner, S. L., Briggs, K. R., et al. 2005, ApJ, 626, L53

Güdel, M., Padgett, D. L., \& Dougados, C. 2006, in Protostars and Planets V, ed. B. Reipurth, D. Jewitt, \& K. Keil, in press, [astro-ph/0609520]

Güdel, M., Briggs, K. R., Arzner, K., et al. 2007, A\&A, 468, 353

Guieu, S., Dougados, C., Monin, J. L., Magnier, E., \& Martín, E. L. 2006, A\&A, 446,485

Hong, J., Schlegel, E. M., \& Grindlay, J. E. 2004, ApJ, 614, 508

Imanishi, K., Tsujimoto, M., \& Koyama, K. 2001, ApJ, 563, 361

Imanishi, K., Nakajima, H., Tsujimoto, M., Koyama, K., \& Tsuboi, Y. 2003, PASJ, 55, 653

Jansen, F., Lumb, D., Altieri, B., et al. 2001, A\&A, 365, L1

Kraft, R. P., Burrows, D. N., \& Nousek, J. A. 1991, ApJ, 374, 344

Leggett, S. K., Allard, F., \& Hauschildt, P. H. 1998, ApJ, 509, 836

Luhman, K. L. 2000, ApJ, 544, 1044

Luhman, K. L. 2004, ApJ, 617, 1216

Luhman, K. L. 2006, ApJ, 645, 676

Luhman, K. L., Whitney, B. A., Meade, M. R., et al. 2006, ApJ, 647, 1180

Martín, E. L. 1998, AJ, 115, 351

Martín, E. L., \& Bouy, H. 2002, New Astron., 7, 595

Martín, E. L., Dougados, C., Magnier, E., et al. 2001, ApJ, 561, L195

Mewe, R., Kaastra, J. S., \& Liedahl, D. A. 1995, Legacy, 6, 16

Mitra-Kraev, U., Harra, L. K., Güdel, M., et al. 2005, A\&A, 431, 679

Mohanty, S., \& Basri, G. 2003, ApJ, 583, 451

Mohanty, S., Jayawardhana, R., \& Basri, G. 2005, ApJ, 626, 498

Mokler, F., \& Stelzer, B. 2002, A\&A, 391, 1025

Morrison, R., \& McCammon, D. 1983, ApJ, 270, 119

Muzerolle, J., Luhman, K. L., Briceño, C., Hartmann, L., \& Calvet, N. 2005a, ApJ, 625, 906

Muzerolle, J., Megeath, S. T., Flaherty, K. M., et al. 2005b, ApJ, 620, L107

Nakajima, T., Oppenheimer, B. R., Kulkarni, S. R., et al. 1995, Nature, 378, 463

Neuhäuser, R., \& Comerón, F. 1998, Science, 282, 83

Neuhäuser, R., Briceño, C., Comerón, F., et al. 1999, A\&A, 343, 883

Ozawa, H., Grosso, N., \& Montmerle, T. 2005, A\&A, 429, 963

Peres, G., Orlando, S., \& Reale, F. 2004, ApJ, 612, 472

Pizzolato, N., Maggio, A., Micela, G., Sciortino, S., \& Ventura, P. 2003, A\&A, 397, 147

Preibisch, T., \& Zinnecker, H. 2001, AJ, 122, 866

Preibisch, T., \& Zinnecker, H. 2002, AJ, 123, 1613

Preibisch, T., Kim, Y.-C., Favata, F., et al. 2005a, ApJS, 160, 401

Preibisch, T., McCaughrean, M. J., Grosso, N., et al. 2005b, ApJS, 160, 582

Rebolo, R., Zapatero-Osorio, M. R., \& Martin, E. L. 1995, Nature, 377, 129

Rieke, G. H., \& Lebofsky, M. J. 1985, ApJ, 288, 618

Rutledge, R. E., Basri, G., Martín, E. L., \& Bildsten, L. 2000, ApJ, 538, L141

Schmitt, J. H. M. M., \& Liefke, C. 2002, A\&A, 382, L9

Siess, L., Dufour, E., \& Forestini, M. 2000, A\&A, 358, 593

Slesnick, C. L., Hillenbrand, L. A., \& Carpenter, J. M. 2004, ApJ, 610, 1045

Slesnick, C. L., Hillenbrand, L. A., \& Carpenter, J. M. 2005, ApJ, 625, 1063

Stelzer, B. 2004, ApJ, 615, L153

Stelzer, B., Micela, G., \& Neuhäuser, R. 2004, A\&A, 423, 1029

Stelzer, B., Micela, G., Flaccomio, E., Neuhäuser, R., \& Jayawardhana, R. 2006, A\&A, 448, 293

Strüder, L., Briel, U., Dennerl, K., et al. 2001, A\&A, 365, L18

Townsley, L. K., Broos, P. S., Nousek, J. A., \& Garmire, G. P. 2002, Nuclear Instruments and Methods in Physics Research A, 486, 751

Tsuboi, Y., Maeda, Y., Feigelson, E. D., et al. 2003, ApJ, 587, L51

Turner, M. J. L., Abbey, A., Arnaud, M., et al. 2001, A\&A, 365, L27

Vuong, M. H., Montmerle, T., Grosso, N., et al. 2003, A\&A, 408, 581

Weisskopf, M. C., Brinkman, B., Canizares, C., et al. 2002, PASP, 114, 1

Wichmann, R., Krautter, J., Schmitt, J. H. M. M., et al. 1996, A\&A, 312, 439

Wilking, B. A., Greene, T. P., \& Meyer, M. R. 1999, AJ, 117, 469 
N. Grosso et al.: X-ray emission from the young brown dwarfs of the Taurus molecular cloud, Online Material $p 1$

\section{Online Material}




\section{Appendix A: Log and data reduction of Chandra observations}

Table A.1 gives the log of Chandra observations which surveyed serendipitously the TMC BDs, and used to supplement the XEST.

Data reduction of archival Chandra data were performed with CIAO software package version 3.2.1, XSPEC version 11.3, the Penn State charge transfer inefficiency (CTI) corrector version 1.45 , and the acis_extract package version $3.67^{10}$. We followed Penn State University (PSU) procedures (e.g., Getman et al. 2005) starting from data reduction from Level 1 event files provided by the Chandra X-Ray Center (CXC). We used the PSU CTI Corrector (Townsley et al. 2002) to correct partially the data for CCD CTI caused mainly by radiation damage at the beginning of the Chandra mission. Source detections were performed with the CIAO's task wavdetect. 2MASS counterparts were used to correct small boresight errors. The acis_extract package was then used to extract source photons, estimate local background, construct source and background spectra, compute redistribution matrix files (RMFs) and auxiliary response files (ARFs), construct light curves and time-energy diagrams, and perform automated spectral grouping and fitting.

\section{Appendix B: Quiescent X-ray light curves of the TMC BDs}

Figure B.1 shows the X-ray light curves of the brightest TMC BDs, which are consistent with quiescent activity. Figure B.1 shows X-ray light curves of BDs observed at two different epochs.

\section{Appendix C: Degeneracy of the quantile diagram}

In contrast to the continuum models used as illustration of quantile diagram by Hong et al. (2004), which connect one single couple of physical parameters to unique X-ray colours, we found that the spectra of optically thin plasma (i.e. a continuum bremsstrahlung plus emission lines) may have several couple of physical parameters producing the same X-ray colours. To use the quantile analysis safely, we need to reckon for each physical parameter the number of possible values per X-ray colour.

We divided first the quantile diagram in a regular grid with a $0.03 \times 0.03$ resolution. Then, the uneven grid of $X$-ray colours of each physical parameter was projected to this regular grid using spline interpolation. Each physical parameter therefore generates a 3-dimensional surface regularly sampled in the X-ray colour plane. Finally, we found numerically the number of intersections between this surface and a line of constant colour, i.e. the degeneracy level.

Figures C. 1 and C.2 show the result for the hydrogen column density and the plasma temperature grids, respectively, computed with WABS $\times$ MEKAL plasma model with 0.3 times the solar elemental abundances and XMM-Newton EPIC pn RMF and ARF. The left pannels show the degeneracy maps, which are nearly identical when one considers the uneven sampling of the model parameter; in Fig. 5 we plotted conservatively the maximum of these two degeneracy maps. The right pannels show for each parameter the corresponding 3-dimensional surface in the X-ray colour plane with folds producing multiple solutions.

\footnotetext{
10 Descriptions and codes for CTI correction and acis_extract can be found at http://www.astro.psu.edu/users/townsley/cti and http://wWw . astro.psu.edu/xray/docs/TARA, respectively.
}

The quantile diagram is mainly composed of areas where X-ray colours correspond to a unique couple of parameters, but areas with multiple solutions - from 2 to 5 - are also present. However, the area surface decreases with the number of multiple solutions.

Figure C. 3 shows an illustration of degenerated solutions if $y=1.3$. For example if $x_{1} \leq x \leq x_{2}$ there is a unique solution for $N_{\mathrm{H}}$ and $k T$. If $x_{2} \leq x \leq x_{3}$ there is a double solution: a first solution with low $N_{\mathrm{H}}$ and high $k T$, and a second solution with high $N_{\mathrm{H}}$ and low $k T$. Generally speaking, if there are $N$ solutions of $N_{\mathrm{H}}$ and $k T$ ordered by increasing values, the solution number $n$ (with $n$ ranging from 1 to $N$ ) is defined as the $n$ th-value of $N_{\mathrm{H}}$ and the $N+1-n$ th-value of $k T$. Degeneracy on both parameter can then be fixed using extra knowledge on only one parameter.

We used the knowledge of the optical extinction to derive the value of the hydrogen column density using the relation $N_{\mathrm{H}}=1.6 \times 10^{21} A_{\mathrm{V}} \mathrm{cm}^{-2} \mathrm{mag}^{-1}$ (Vuong et al. 2003; Cardelli et al. 1989). The best estimate of the temperature is then the temperature solution corresponding to the hydrogen column density solution which is the closest to this value. 
N. Grosso et al.: X-ray emission from the young brown dwarfs of the Taurus molecular cloud, Online Material p 3

Table A.1. Archival Chandra observations which surveyed serendipitously the TMC BDs. Column (1) gives the name of the instrument used: "I" $\left(17^{\prime} \times 17^{\prime}\right.$ field of view) and "S" $\left(8.5^{\prime} \times 25.5^{\prime}\right.$ field of view) for imaging and spectroscopy ACIS CCD (Garmire et al. 2003), respectively. Column (8) give the name of the TMC BD whitin the Chandra field of view. Column (9) indicates whether we use this archival data to supplement the XEST or not (see following notes). Notes: in the ACIS-S observation \#3364, the TMC BD KPNO-Tau 2 was located 13' off-axis on ACIS-S2, and it is not detected; we obtained here a better constraint on the X-ray luminosity of this BD using the sum of the two XMM-Newton exposures, rather than this ACIS-S observation. In the ACIS-S observation \#4488, the TMC BD 2MASS J0421 was located 5.7' off-axis on ACIS-S3, the pipeline detection algorithm found no source at this location.

\begin{tabular}{|c|c|c|c|c|c|c|c|c|}
\hline$\overline{\overline{\text { ObsID }}}$ & "ACIS- & $\overline{\text { Target }}$ & $\overline{\text { Nomina }}$ & $\overline{\overline{\text { ointing }}}$ & Start/End obs. & Exposure & TMC BD & Used \\
\hline (1) & (2) & (3) & $\begin{array}{c}\alpha_{\mathrm{J} 2000} \\
(4)\end{array}$ & $\begin{array}{c}\delta_{\mathrm{J} 2000} \\
(5)\end{array}$ & (6) & $\begin{array}{l}\text { ks } \\
\text { (7) }\end{array}$ & (8) & (9) \\
\hline 1866 & I & L1551 & $04^{\mathrm{h}} 31^{\mathrm{m}} 32^{\mathrm{s}} .7$ & $18^{\circ} 08^{\prime} 08^{\prime \prime}$ & 2001-07-23T05:10:11/24T03:52:12 & 79 & MHO 4 & yes \\
\hline 2563 & I & L1527 & $04^{\mathrm{h}} 39^{\mathrm{m}} 52^{\mathrm{s}} .7$ & $26^{\circ} 03^{\prime} 05^{\prime \prime}$ & 2002-12-06T08:30:12 / 06T14:21:15 & 20 & CFHT-BD-Tau 4 & yes \\
\hline 3364 & $\mathrm{~S}$ & V410 Tau & $04^{\mathrm{h}} 18^{\mathrm{m}} 34^{\mathrm{s}} .6$ & $28^{\circ} 22^{\prime} 47^{\prime \prime}$ & 2002-03-07T06:16:32 / 07T11:45:24 & 18 & KPNO-Tau 2 & no \\
\hline 4488 & $\mathrm{~S}$ & FS Tau & $04^{\mathrm{h}} 22^{\mathrm{m}} 00^{\mathrm{s}} .1$ & $26^{\circ} 58^{\prime} 07^{\prime \prime}$ & 2003-11-08T12:57:58 / 08T21:56:34 & 30 & 2MASS J0421 & no \\
\hline
\end{tabular}
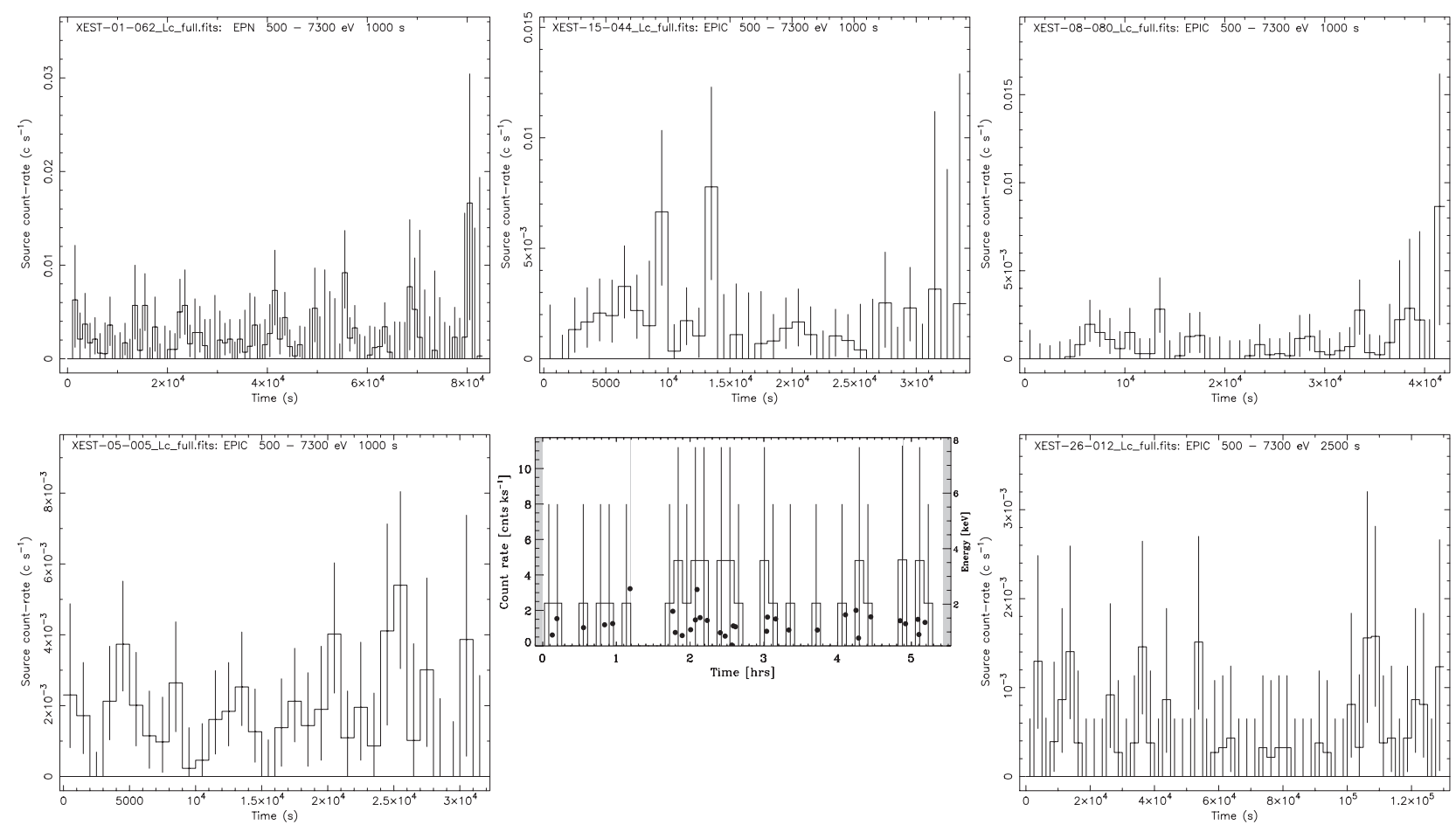

Fig. B.1. X-ray light curves of BDs in the TMC. From top to bottom and left to right: 2MASS J0422 (only pn data), KPNO-Tau 5, CFHT-BD-Tau 3, CFHT-Tau 6 (EPIC), CFHT-BD-Tau 4 (ACIS-I), and 2MASS J0455 (only MOS data). On the Chandra light curve, black dots indicate the arrival time and energy of individual X-ray photons. These light curves are consistent with quiescent emission. 
N. Grosso et al.: X-ray emission from the young brown dwarfs of the Taurus molecular cloud, Online Material p 4
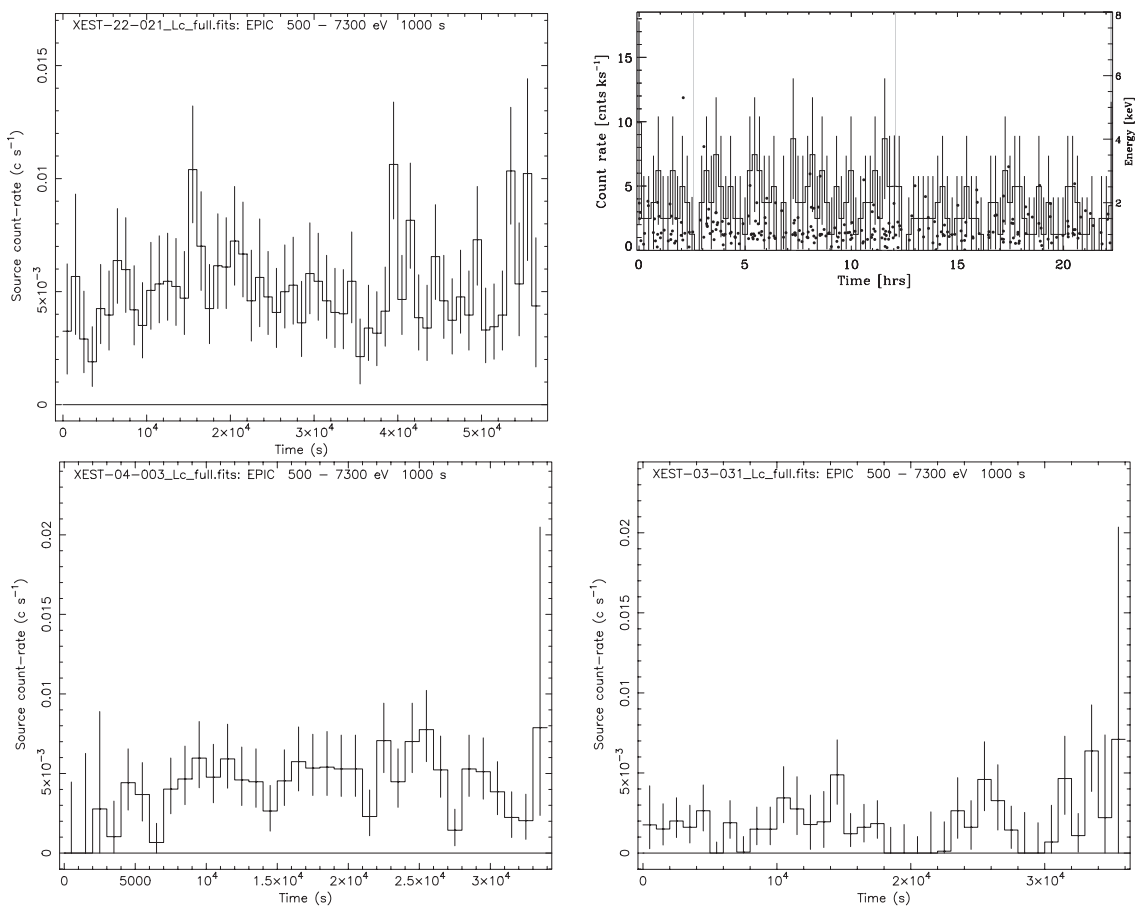

Fig. B.2. X-ray light curves of BDs in the TMC observed at two different epochs. Top and bottom row show MHO 4 and CFHT-Tau 5 light curves, respectively. During the second XMM-Newton observation of CFHT-Tau 5, the source is located on an EPIC pn gap, which explains the count rate difference observed between the two epochs. 
N. Grosso et al.: X-ray emission from the young brown dwarfs of the Taurus molecular cloud, Online Material p 5
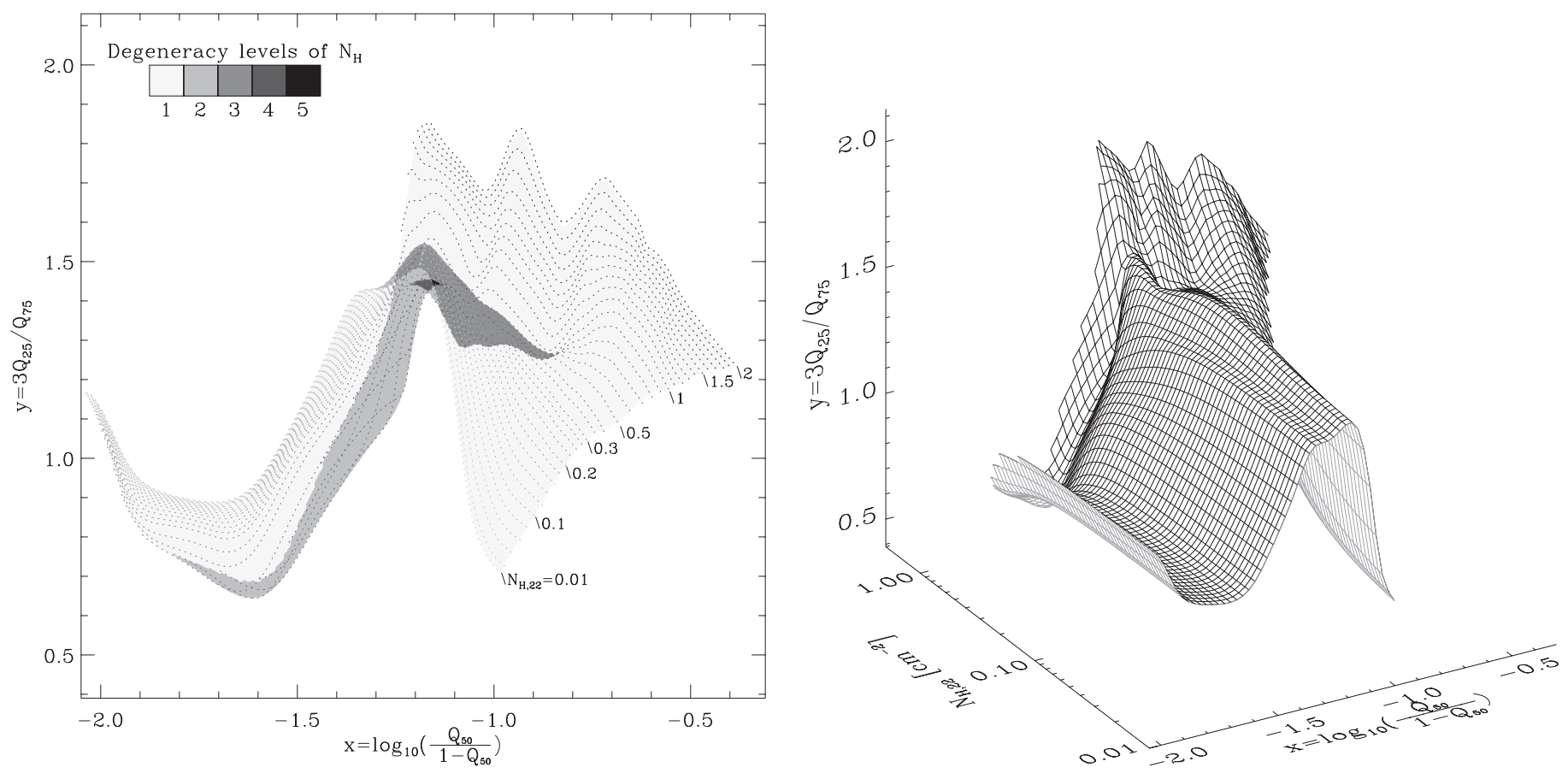

Fig. C.1. Degeneracy map of the absorption column density parameter $\left(N_{\mathrm{H}}\right)$ in the quantile diagram. Left: quantile diagram showing in the $(x, y)$ space colours the loci of constant $N_{\mathrm{H}}$ values (dotted lines) for a WABS $\times$ MEKAL plasma model with 0.3 times the solar elemental abundances using $X M M$-Newton EPIC pn RMF and ARF. Grey levels indicate for each $(x, y)$ value the number of corresponding absorption column density values. Regions where a $(x, y)$ value correspond to a unique value of $N_{\mathrm{H}}$ are coloured in light grey. Right: 3-dimensional shape of the surface generated by $N_{\mathrm{H}}$ from the colour space of the quantile diagram $(x, y)$. Folds produce the degeneracy observed in the quantile diagram.
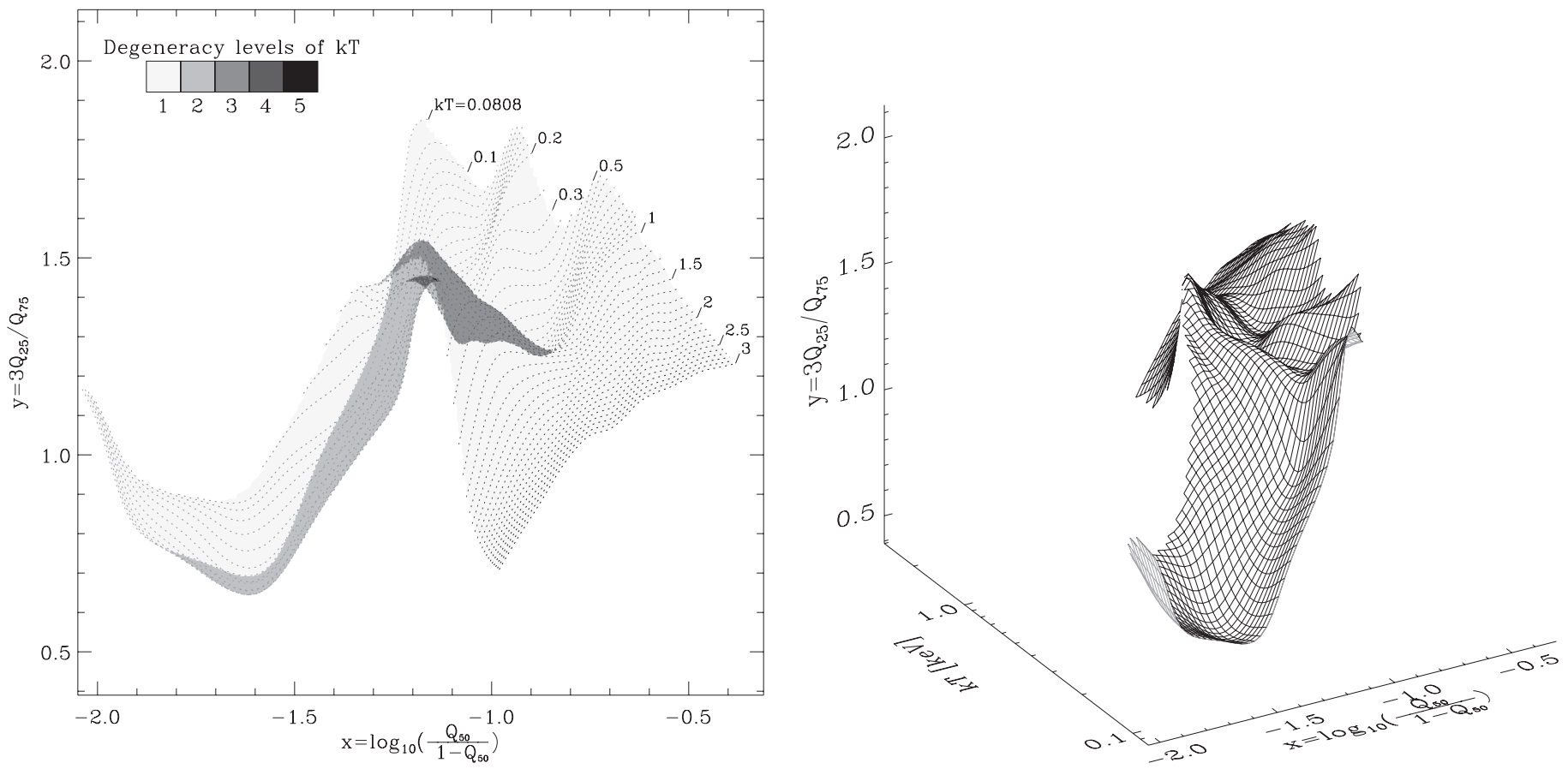

Fig. C.2. Degeneracy map of the plasma temperature parameter $(k T)$ in the quantile diagram. Left: quantile diagram showing in the $(x, y)$ space colours the loci of constant $k T$ values (dotted lines) for a WABS $\times$ MEKAL plasma model with 0.3 solar elemental abundance using XMMNewton EPIC pn RMF and ARF. Grey levels indicate for each $(x, y)$ value the number of corresponding plasma temperature values. Regions where a $(x, y)$ value correspond to a unique value of $k T$ are coloured in light grey. Right: 3D shape of the surface generated by $k T$ from the parameter space of the quantile diagram $(x, y)$. Folds produce the degeneracy observed in the quantile diagram. 
N. Grosso et al.: X-ray emission from the young brown dwarfs of the Taurus molecular cloud, Online Material p 6
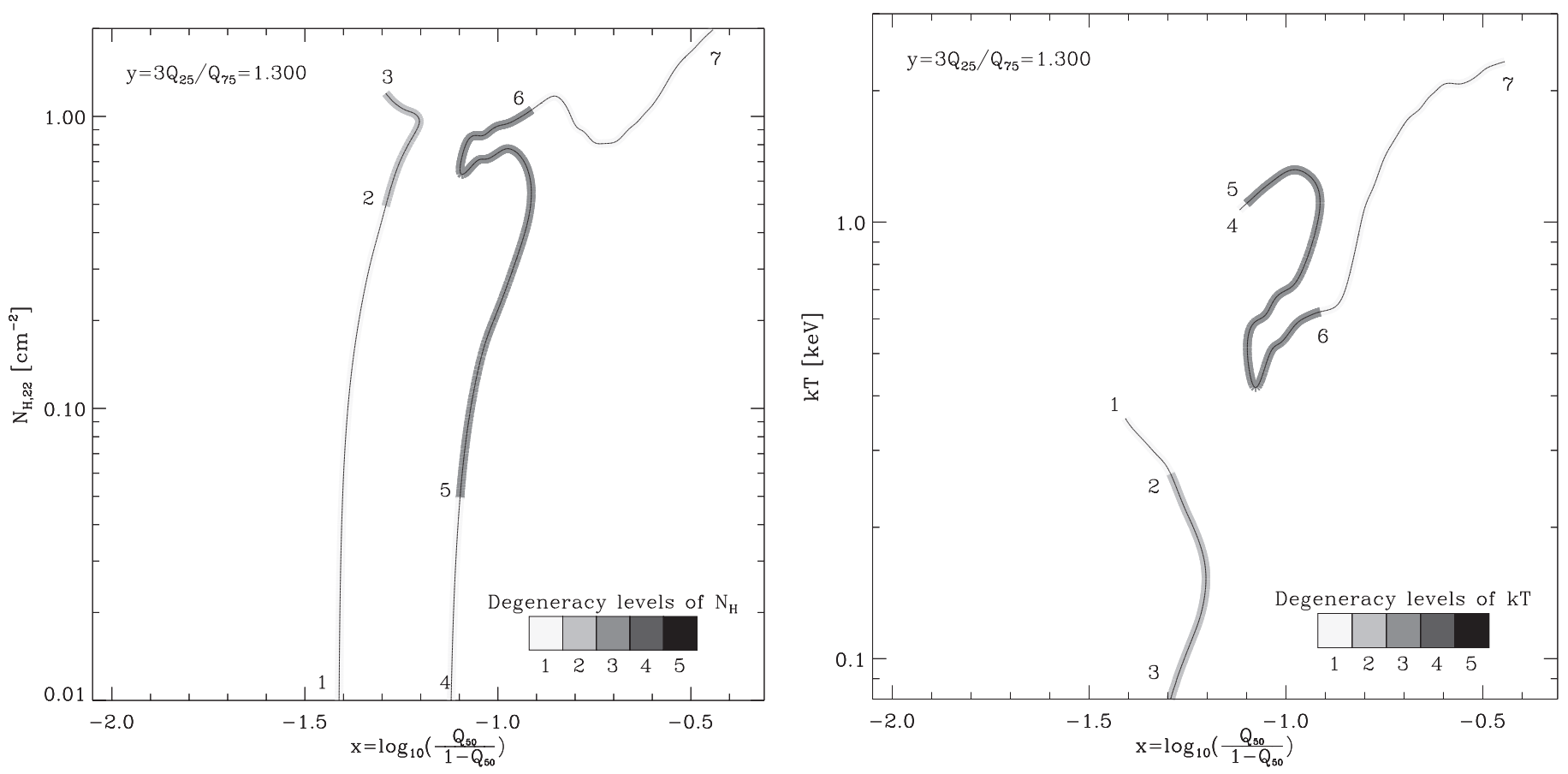

Fig. C.3. Illustration of degenerated solutions. Left: horizontal cut of the $N_{\mathrm{H}}$ surface (Fig. C.1 right) at $y=1.3$. Right: horizontal cut of the $k T$ surface (Fig. C.2 right) at the same elevation. The line colour indicates the degeneracy level of the parameter. Numbers label the boundary/changing points when one moved on these surfaces along the $x$ direction, and give the correspondance between the two parameters. 